\title{
Antigal Motaite: una aldea temprana en Abdón Castro Tolay (Barrancas), Puna de Jujuy
}

\section{Rodolphe Hoguin}

Instituto Interdisciplinario de Ciencias Básicas (ICB), Laboratorio de Paleoecología Humana, Facultad de Ciencias Exactas y Naturales, Universidad Nacional de Cuyo (UNCu) y Consejo Nacional de Investigaciones Científicas y Técnicas (CONICET). Padre Jorge Contreras 1300, Parque General San Martín (CP 5300), Mendoza, Argentina. E-mail: rhoguin@mendoza-conicet.gob.ar

\section{Patricia Solá}

\author{
(iD) https://orcid.org/0000-0002-3608-7967 \\ Instituto de Arqueología, Facultad de Filosofía y Letras, Universidad de Buenos Aires (UBA) - \\ Consejo Nacional de Investigaciones Científicas y Técnicas (CONICET). 25 de mayo $217,3^{\circ}$ piso \\ (CP C1002ABE), Ciudad Autónoma de Buenos Aires, Argentina. E-mail: patriciasola@gmail.com

\section{Hugo D. Yacobaccio} \\ (iD) https://orcid.org/0000-0002-8211-3978 \\ Instituto de Arqueología, Facultad de Filosofía y Letras, Universidad de Buenos Aires (UBA) - Consejo \\ Nacional de Investigaciones Científicas y Técnicas (CONICET). 25 de mayo $217,3^{\circ}$ piso (CP C1002ABE), \\ Ciudad Autónoma de Buenos Aires, Argentina. E-mail: hdyacobaccio@gmail.com
}

\section{Resumen}

El sitio Antigal Motaite, emplazado en las vecindades de la localidad Abdón Castro Tolay (Barrancas, Puna de Jujuy), es un conglomerado constituido por unas 37 estructuras de formas cuadrangulares y circulares. Dos fechados radiocarbónicos permiten ubicar este sitio ca. 1800-1700 años AP. Durante el Período Temprano (3000-1000 años AP), el desarrollo de la agricultura empieza a tener mayor importancia y, al mismo tiempo, se evidencia una disminución de la movilidad regional de las poblaciones pastoriles. En esta ocasión, se presentan los primeros resultados de las excavaciones realizadas en este antiguo poblado. Se incluye también un relevamiento preliminar de la arquitectura y un primer análisis de las tecnologías lítica y cerámica. Los datos obtenidos, comparados con los antecedentes arqueológicos regionales, evidencian una ocupación agropastoril estable y sedentaria, constituida probablemente por varias unidades familiares. La ocupación se habría caracterizado por llevar adelante estrategias domésticas de subsistencia, trayectorias tecnológicas especializadas y el aprovisionamiento de bienes alóctonos obtenidos mediante el tráfico caravanero. En este contexto, se ha detectado la introducción de varios cambios tecnológicos referidos a la producción cerámica y lítica. 


\title{
Antigal Motaite: an early village in Abdón Castro Tolay (Barrancas), Jujuy Puna
}

\begin{abstract}
The Antigal Motaite site, located in the vicinity of the Abdón Castro Tolay locality (Barrancas, Puna of Jujuy), is a conglomerate consisting of about 37 structures of quadrangular and circular shapes. Two radiocarbon dates allow us to locate this site ca. 1800-1700 years BP. During the Early Period (3000-1000 BP), the development of agriculture became important. At the same time, there is evidence of a decrease in the regional mobility of pastoral populations. In this paper, we present the first results of the excavations carried out in this old village. A preliminary survey of the architecture and a first analysis of the lithic and ceramic technologies are also included. Compared to the regional archaeological knowledge, the data obtained show a stable and sedentary agropastoral occupation, probably constituted by several family units. This occupation was characterized by domestic subsistence strategies, specialized technological trajectories, and the supply of allochthonous goods obtained through caravan traffic. In this context, the introduction of several technological changes related to ceramic and lithic production has been detected.
\end{abstract}

KEY-WORDS: Early Period, Lithic and ceramic technology, Domestic strategies, Jujuy Puna.

\section{Introducción}

La formación de asentamientos conglomerados constituye uno de los mayores cambios de las sociedades humanas. El surgimiento de estos poblados ha sido interpretado como resultado de la sedentarización o de una reducción de la movilidad de los grupos humanos. A su vez, su presencia respondería a una organización social distinta a la de poblaciones cazadoras-recolectoras de alta movilidad residencial, alcanzada a través de la especialización y de la intensificación de las estrategias de subsistencia. La adopción de la agricultura y del pastoreo en los Andes Centro-Sur funcionó como un detonante para que se diera este proceso (Olivera, 2012; Tarragó, 1984). En la región, aún no se ha definido cabalmente la fecha más temprana de estas primeras aldeas. Una excepción cronológica para este tipo de asentamientos sería el caso de los sitios Tulán 52 y 54 , en el sector oriental del Salar de Atacama (Chile), con fechados coincidentes con el final del Holoceno medio en T52 (ca. 4800-3600 años AP). Además, en Tulán 52 , los estudios zooarqueológicos corroboraron el desarrollo de una subsistencia fundamentada en recursos silvestres y domesticados. En Tulán 54, se evidenció una funcionalidad basada principalmente en actividades rituales y estacionales (Cartagena, Núñez y Grosjean, 2007; Núñez y Perlès, 2018; Núñez et al., 2016). En la vertiente argentina, existen algunos hallazgos aldeanos similares con cronologías en torno a 3600 años AP (Muscio, 2011). No obstante, la mayoría de estos asentamientos, generalmente ubicados en fondos de cuenca y con fácil acceso al agua, fue datada entre 2500 y 1500 años AP (De Feo, 2015; Fernández Distel, 2001; Muscio, 2004; Olivera, Elías, Pérez y Salminci, 2015; Raffino, 1977). La emergencia de estos poblados estaría relacionada, principalmente, con el crecimiento poblacional y con una dinámica de intensificación del nicho económico (Muscio, 2011; Muscio y López, 2016).

Los procesos demográficos y poblacionales involucrados en la constitución de las primeras aldeas representan un fenómeno social, económico y cultural de gran amplitud. Estos procesos fueron concomitantes con cambios tecnológicos (Muscio, 2011,2012), pero también con las estrategias de obtención de recursos. El impacto de 
la agricultura entre los 2000 y 1500 años AP así como su interacción con el pastoreo de camélidos, que también se manifestó en los aspectos sociales y económicos de las poblaciones humanas, motivaron cambios en la organización de los sitios (Delfino, Espiro y Díaz, 2007; Grant y Olivera, 2016; Olivera, 2012).

El objetivo general de esta investigación es caracterizar la producción tecnológica y el tipo de asentamiento identificado en la aldea Antigal Motaite durante finales del Período Temprano (3000-1000 años AP) y aportar evidencias a la discusión regional sobre la formación y el desarrollo de las primeras aldeas en la Puna argentina. Los resultados presentados han sido obtenidos a partir del estudio de varias líneas de evidencia, como fechados radiocarbónicos, arquitectura, tecnología lítica, análisis de obsidiana por fluorescencia de Rayos X (FRX) y análisis petrográfico de pastas cerámicas. En particular, se busca determinar cómo se reflejaron los procesos económicos y sociales mencionados en los patrones de asentamiento y en las estrategias de producción tecnológica. Se propone realizar una reconstitución de las cadenas operativas, así como de sus aspectos técnicos y económicos. Se intentará relacionar e interpretar las cadenas operativas a partir del contexto arqueológico regional y del patrón de asentamiento local.

\section{El área de estudio y aspectos paleoambientales}

El pueblo Abdón Castro Tolay -Barrancas- (ca. 3600 m s.n.m.) se ubica pocos kilómetros al noroeste de Salinas Grandes, en el departamento Cochinoca (Jujuy). La localidad fue declarada Reserva Natural y Cultural Municipal debido a la gran riqueza de pinturas y grabados rupestres. El área cuenta con más de 40 sitios con representaciones de ese tipo que ponen de relieve su valor patrimonial. Hasta el presente, se han relevado en esos sitios 143 paneles con más de 1400 motivos plasmados en aleros, cuevas y paredones ignimbríticos que se extienden por varios kilómetros sobre ambas márgenes del río Barrancas.

El área de estudio se encuadra en la Puna Seca de los Andes Centro Sur (Figura 1). La Puna es una meseta de altura (por encima de los $3500 \mathrm{~m}$ s.n.m) ubicada en la cordillera de los Andes, entre los $22^{\circ}$ y $27^{\circ}$ de latitud Sur. Se caracteriza por un clima particularmente frío y seco con precipitaciones que oscilan entre 80 y $200 \mathrm{~mm}$ anuales. El ambiente es fragmentado y presenta variabilidad en cuanto a la distribución de los recursos. La biomasa disponible se agrupa en zonas de concentración de nutrientes, restringidas y cambiantes a través del tiempo (Buitagro y Larran, 1991; Morales, 2011; Yacobaccio, 1994). Es imprescindible aclarar que los camélidos son oriundos de este piso ecológico (aunque no exclusivamente), mientras que varias especies vegetales y animales evidenciadas en el registro arqueológico son alóctonas. Estas interacciones fluctuaron considerablemente en intensidad y en el espacio, pero se mantuvieron en el tiempo. Estos aspectos son de importancia para la constitución y el desarrollo de las economías productivas (pastoreo y agricultura).

Barrancas es una pequeña cuenca endorreica ubicada en una depresión tectónica que es parte de una depresión mayor, cuyo sector meridional está ocupado por las Salinas Grandes y la Laguna de Guayatayoc. Los registros geológicos indican que los procesos erosivos actuantes en el área fueron intensos. Si bien el canal central del río Barrancas está activo en la actualidad, los estudios paleoambientales del área, en base a distintos proxies (diatomeas, polen, carbonatos, MO, susceptibilidad magnética y geomorfología), sugieren que el río evolucionó desde un sistema fluvial permanente hasta uno efímero, pero mantuvo condiciones relativamente húmedas y estables en un marco regionalmente más árido (Morales et al., 2018; Oxman et al., 2019; Pirola et al., 2018). Sin embargo, durante el Holoceno tardío, se pudieron detectar condiciones húmedas, aunque algo inestables, con eventos torrenciales intercalados con episodios 


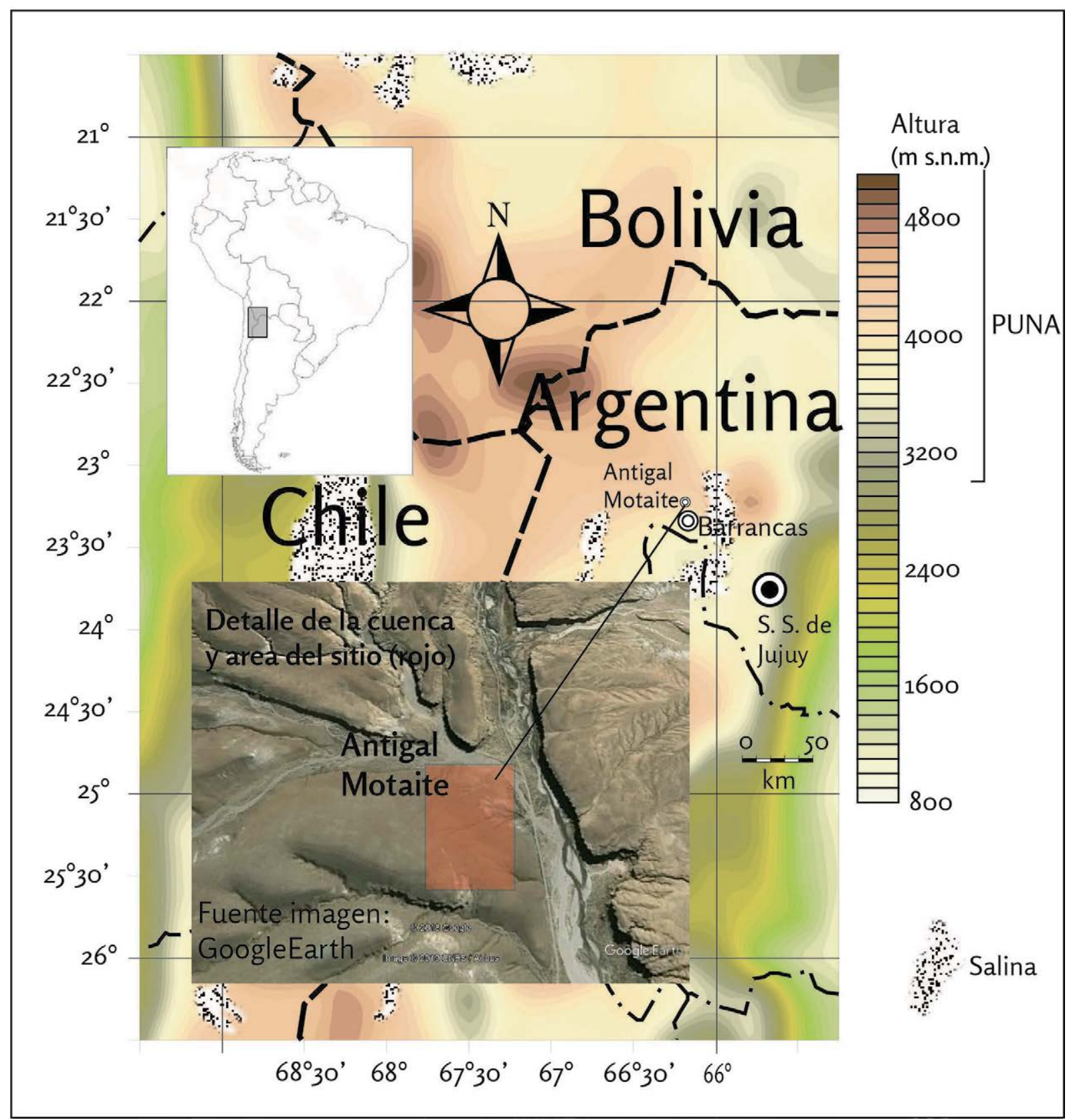

Figura 1. Mapa de localización del área de estudio y del sitio Antigal Motaite.

de pérdida de energía hídrica (Oxman et al., 2019; Pirola et al., 2018). Los análisis polínicos muestran indicadores de perturbación antrópica en las vegas, pudiendo estar relacionados con el impacto de actividades pastoriles a partir del Holoceno tardío, que se habrían intensificado con posterioridad a $2080 \pm 80$ años AP. También se han observado algunas especies indicadoras de humedad local (e.g. Pteridophytas), particularmente, entre 1730 y 1280 años AP (Oxman et al., 2019).

\section{Arqueología de las aldeas tempranas}

La emergencia de los primeros poblados es un extenso tema de discusión que integra gran variabilidad de procesos y de líneas de evidencia. Es importante destacar 
que una aldea, por lo general, se relaciona con la agrupación de varias unidades residenciales que se corresponden con un grupo extendido que denota cierto grado de sedentarismo. La permanencia de tal grupo en este tipo de asentamientos requiere el desarrollo de estrategias de subsistencia que implican una explotación intensiva y complementaria de recursos (Olivera, 2012). La producción de excedentes alimenticios y su almacenaje pueden ser contingencias favorables en este contexto, ya que permitirían un abastecimiento de recursos estacionales para una base residencial de tipo más permanente. Sin embargo, no siempre estas prácticas tienen visibilidad arqueológica, ni tampoco son estrategias imprescindibles en este escenario. De hecho, varios autores interpretaron que las aldeas tempranas eran el resultado de una organización con núcleos domésticos relativamente autónomos (Quesada, 2010; Salazar y Franco Salvi, 2015). Sin embargo, no se descarta la importancia del carácter ceremonial de ciertas aldeas en el desarrollo de estos procesos (Núñez y Perlès, 2018; Núñez et al., 2016).

A partir de 2500 años AP, las ocupaciones puneñas se caracterizan de manera integrada por presentar una economía de tipo productivo, de base predominantemente pastoril y con agricultura complementaria (Albeck, 2010; Núñez y Dillehay, 1979; Olivera, 2012). En este caso, el sedentarismo está asociado a una movilidad logística que genera una distribución dispersa en el espacio con bases residenciales aglutinadas, relativamente permanentes, y ocupaciones temporarias o estacionales, ya sea en puestos pequeños o cuevas y aleros (Olivera, 2012; Yacobaccio et al., 2011). El acceso a ciertos recursos de otros pisos ecológicos, y la circulación de bienes, conocimientos y personas, fue asegurado mediante caravaneo de llamas en un sistema de intercambio regular, aunque no muy intenso (Yacobaccio, 2012). Las ocupaciones pastoriles más antiguas se encuentran principalmente en aleros a partir de ca. 3200-2200 años AP y, hacia el final de este período, aparecen los primeros aglutinamientos de estructuras de piedra, aunque los aleros nunca dejan de ser utilizados (Aschero, 1979; Fernández Distel, 1998; López, 2008; Yacobaccio et al., 2011, entre otros). Estos conglomerados están ubicados preferentemente cercanos a vegas de pastura y ríos, y se encuentran generalmente en quebradas y en fondos de cuenca (De Feo, 2015). Se puede mencionar el surgimiento de manera aislada y temprana de este tipo de estructuras, como sería el caso de Ramadas (Puna de Salta) durante los inicios del Holoceno tardío, ca. 3600 años AP (Muscio, 2011). A medida que se avanza cronológicamente en el Holoceno tardío, se observa mayor concentración de construcciones, jerarquización de los espacios y aumento del tamaño de los sitios (Delfino et al., 2007; Korstanje, 2007; Olivera, 2012). A su vez, estos cambios han sido interpretados como el producto de una mayor población y de cierto grado de organización supra-comunitaria que estimuló el desarrollo de la tecnología agrícola (Quesada, 2010). En este sentido, es importante destacar un importante incremento de la señal antropogénica a partir de ca. 1700 años AP, hecho que representaría un crecimiento poblacional que se manifiesta a partir de un aumento de sitios y localizaciones ocupadas (Muscio y López, 2016).

La agricultura parece haberse iniciado por grupos domésticos y fue seguida por un crecimiento de población, una estandarización de la producción y por la aparición de centros habitacionales construidos con cierto grado de planificación (Tchilinguirian y Olivera, 2010). Hacia ca. 2000 años AP, este tipo de organización parece haber tenido mayor incidencia en las estrategias de las sociedades pastoriles tempranas, con ocupaciones más intensas y permanentes (Olivera et al., 2015). En la Puna, la producción agrícola se habría desarrollado de manera complementaria al pastoreo, y debió de ser diversificada y de muy baja escala, adoptando, muy posiblemente, una estrategia de tipo rotativo. A lo largo de los dos últimos milenios, se produjeron cambios en las prácticas agrícolas y en la intensidad de la producción que fueron acompañados por varias innovaciones tecnológicas (palas, azadas, acequias y canales de riego, etc.) (Quesada, 2010). 
En Antofagasta de la Sierra y a partir de 1800 años AP, la incorporación de maíz como forraje marcó un cambio sustancial en las estrategias pastoriles; el resultado fue el abandono de la trashumancia y la mayor permanencia de las poblaciones en torno a las bases residenciales (Grant y Olivera, 2016). Asimismo, fue notable la interacción complementaria entre las dos estrategias de producción, el pastoreo y la agricultura, ya que el guano también podía ser utilizado como fertilizante. De este modo, una agricultura más intensiva y extensiva en relación con cronologías anteriores (ver Babot, 2011; Olizewski y Olivera, 2009) se consolidaría en esta región en torno a 1000 años AP (Tchilinguirian y Olivera, 2010).

Una de las innovaciones tecnológicas de gran interés es la cerámica cuyo surgimiento en la macro región se produjo en torno a los 3000 años AP (Fernández Distel, 1998; González, 2004; Olivera, 2012, entre otros). Existen registros de hallazgos aislados de cerámica anteriores a esa fecha, por ejemplo, PCh1.5, datado ca. 3800 años AP (Aschero y Hocsman, 2011), o Ramadas con ca. 3600 años AP (Muscio, 2011). A partir de 3200 años $A P$, la cerámica experimenta una expansión notable acompañada de importante diversidad de formas y decoraciones; además, esta tecnología cumple un rol fundamental tanto en lo económico y social como en lo simbólico, llegando a constituir el principal mobiliario funerario del período (Olivera, 2012). La cerámica marcó un cambio importante en las actividades de almacenaje y cocción de alimentos, e incidió notablemente en las prácticas sociales, los modos de producción y la distribución de los recursos.

Los cambios tecnológicos líticos registrados en el Período Temprano fueron observados en varias regiones. Es factible que, en parte, estuviesen relacionados con la introducción del arco y la flecha. En relación con este sistema de propulsión y enmangamiento, se puede mencionar el hallazgo de pequeñas puntas triangulares con aletas y pedúnculo, mayormente confeccionadas en obsidiana y vulcanita (s.I.) (Escola, 2002). Se destaca el uso de raederas de módulo grandísimo relacionadas con prácticas específicamente agrícolas a partir de 1700 años AP, lo que pone de relieve, una vez más, el papel de los cultivos en la economía agro-pastoril entre el primer y el segundo milenio AP en Antofagasta de la Sierra (Escola, Hocsman y Babot, 2013). Finalmente, y en relación con la producción agrícola, se debe mencionar el desarrollo de tecnologías hidráulicas con redes de acequias más o menos complejas para el riego de los cultivos (Korstanje, Quesada, Franco Salvi, Lema y Maloberti, 2015, Quesada, 2010).

\section{Materiales y métodos}

En Antigal Motaite se realizó un relevamiento planimétrico de las estructuras del sitio y se midió su largo máximo. La unidad arquitectónica de análisis comprende el recinto o el segmento de muro aislado o articulando diferentes estructuras. En este trabajo, se presentan las estadísticas univariadas del largo máximo. Asimismo, se describen preliminarmente las técnicas de construcción y su posible funcionalidad en relación con sus dimensiones y su morfología en comparación con otras aldeas. Por otro lado, se efectuaron ocho sondeos. Dado que no se pudieron observar diferencias de composición sedimentaria, se realizaron divisiones estratigráficas basadas en la distribución de hallazgos y remanentes de estructuras. Se publican en este artículo dos fechados $\mathrm{C} 14$ AMS realizados en dos de los sondeos, calibrados mediante el programa CALIB 8.1.0 (Stuiver y Reimer, 1993) y la curva SHCal20 (Hogg et al., 2020). Además, se recolectaron materiales de superficie a partir de cinco transectas, orientadas norte-sur, de donde proviene la mayoría de los materiales analizados. Debido a la notable escasez de restos faunísticos, en esta presentación no ha sido incluida dicha línea de evidencia.

El análisis lítico se enfocó sobre la reconstitución de cadenas operativas (Inizan, Reduron, Roche y Tixier, 1995). En la primera etapa, se clasificaron los artefactos según 
las materias primas. El paso siguiente incluyó la división conceptual entre los artefactos con retoque y aquéllos con formatización (façonnage). Se considera que el retoque ocurre cuando el método de desbaste es predeterminado y produce formas base con algunos de los criterios tecnofuncionales ya presentes en la pieza. Al contrario, cuando los soportes presentan mayor variabilidad y menor predeterminación, la formatización es mayor. Algunos instrumentos, núcleos y formas base fueron graficados según las normativas utilizadas para determinar el sentido, la dirección y el orden de extracción, con el fin de establecer las secuencias de producción (Inizan et al., 1995). También, se clasificaron los núcleos en tres tipos: $\mathrm{C}$ (extracciones aisladas o serie unidireccional de $\sim 3$ formas base), SASP (Sistema alternando superficie y plataforma, produciendo varias series de $\sim 3$ formas base), $D$ otros (varias series multidireccionales de $\sim 3$ formas base) y centrípetos/discoidales. Asimismo, se identificaron las unidades tecno-funcionales (UTF) reconocidas en base a patrones tecnológicos y morfológicos de los filos (Boëda, 2013). En el marco de este trabajo, también se identificaron las UTF prehensivas y transformativas. Las primeras corresponden a características volumétricas aptas para el enmangamiento (pedúnculo, adelgazamiento, etc.) o para una prehensión manual (filos sinuosos embutidos, dorsos altos, etc.), mientras que las segundas presentan rasgos que permiten una transformación del material -cortar, perforar, raspar, ranurar, etc.- en función del tipo de instrumento. Se caracterizaron las relaciones entre UTF y las cadenas operativas. Los términos descriptivos provienen de los trabajos de Inizan et al. (1995) y de Boëda (2013). El objetivo de aplicar esta metodología es diferenciar trayectorias tecnológicas e identificar sus aspectos económicos, funcionales y sociales (Lemonnier, 1986).

La cerámica fue estudiada en dos etapas. Un primer análisis macroscópico fue dirigido a clasificar y establecer parámetros básicos como forma, tamaño y tipo de vasija, técnicas de manufactura, de terminación superficial y técnicas decorativas. La muestra total fue observada bajo microscopio estereoscópico permitiendo clasificar las pastas según los antiplásticos dominantes y seleccionar las muestras que serían estudiadas bajo microscopio petrográfico de polarización. Dicha selección incluyó tiestos representativos de la cerámica ordinaria y algunos fragmentos que, por su decoración o porque representaban formas poco frecuentes (por ej., los fragmentos de pipa), se diferenciaban del conjunto. En esta instancia de la investigación, se tomó como patrón de comparación el resultado del análisis petrográfico realizado sobre los primeros ocho cortes delgados confeccionados en tiestos de recolección superficial. Mediante la determinación de la fracción gruesa (antiplásticos), se establecieron grupos de pastas, lo que permitió inferir el origen de las materias primas utilizadas y discutir su gestión en relación con el tipo de producción.

\section{Antigal Motaite: una aldea del período temprano}

El sitio consiste en un conglomerado de recintos rectangulares, circulares y semicirculares. Hasta el presente, se han registrado 37 estructuras entre las que se destacan algunos recintos rectangulares de gran tamaño (Figura 2). De los ocho sondeos efectuados, cuatro fueron estériles (en materiales cerámicos, líticos y orgánicos) y presentaron una capa compuesta por arena mediano-fina, aunque en algunos de los sondeos y a somera profundidad, se localizaron segmentos de recintos anteriores o contemporáneos. Se puede mencionar la presencia de un mortero que permanece en el lugar (largo máximo: $55,5 \mathrm{~cm}$ ) en la estructura 33 (Figura 2). El sondeo $(1 \times 1 \mathrm{~m})$ interno en la estructura 17, tampoco presentó diferenciación estratigráfica. Entre los bloques y por debajo de los mismos, se hallaron algunos fragmentos cerámicos, un instrumento lítico (tipo rodado tallado), una pequeña costilla de roedor y una cueva de roedor. En el montículo (estructura 17), se relevó un bloque romboidal (eje mayor ca. $45 \mathrm{~cm}$ ) grabado con pequeñas concavidades 


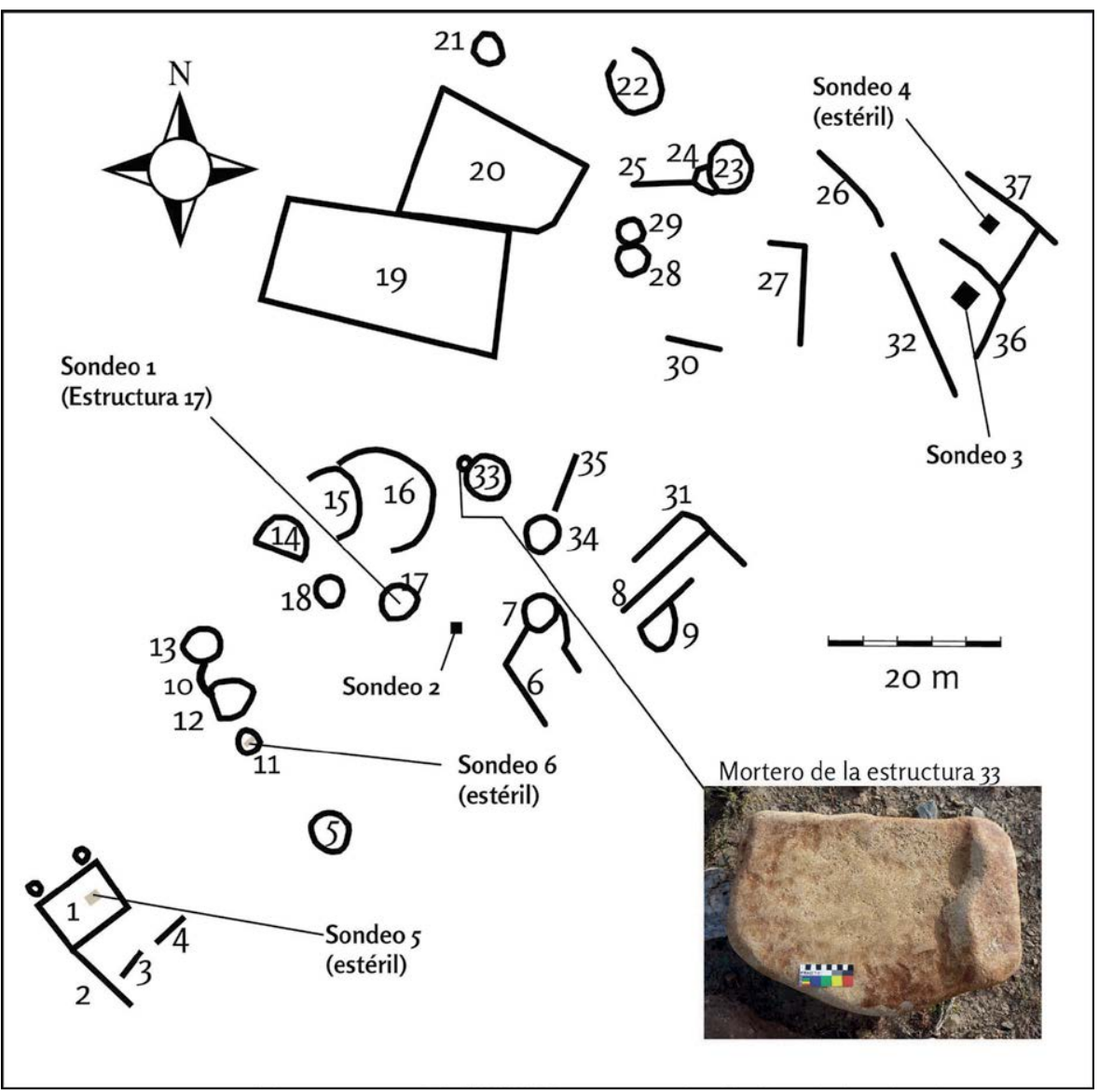

Figura 2. Planta general del sitio y ubicación de los sondeos.

(horadadas) que definen un círculo en su sector más ancho. El sondeo 2 (60 x 60 $\mathrm{cm}$ ) fue realizado al este del montículo, por fuera de la estructura. Tampoco se pudo observar una diferenciación estratigráfica y, en ambos casos, el sedimento está compuesto por arena de granulometría fina a mediana. Sin embargo, en este segundo sondeo se hallaron algunos desechos líticos pequeños y restos óseos en mal estado de preservación. También se registró una lente de ceniza con carbones (profundidad: ca. $40 \mathrm{~cm}$ ). Uno de los carbones permitió obtener el fechado de 1780 \pm 15 años AP [249-342 cal. d.C. $(p=0,93)$ y 351-360 cal. d.C. $(p=0,07)$, calibrado a 2 sigmas, Código de laboratorio: GifA\#16254/ECHo\#1155\&1202].

En el sondeo 3, se detectaron dos posibles ocupaciones. Al igual que en los sondeos descritos previamente, no se pudo establecer ninguna diferenciación sedimentaria; no obstante, en función de la disposición vertical de los materiales y de los segmentos de estructura, se dividió el sondeo en tres extracciones (Figura 3). El sedimento tiene las mismas características que en el resto del sitio, arena fino-mediana, pero se vuelve más heterogéneo a partir de los $11 \mathrm{~cm}$ donde algunos componentes alcanzan el tamaño grava. En la capa $1 \mathrm{~A}$, comienzan a registrarse algunos bloques de ignimbrita junto con materiales líticos, cerámicos y faunísticos. A partir de los $20 \mathrm{~cm}$, los bloques son más numerosos y parecen formar una estructura (capa 1B). Los cimientos de los bloques se encuentran a mayor profundidad, cercana a los $36 \mathrm{~cm}$ (capa 1C). En esta capa, se registró un tiesto de cerámica negra junto a una lente de cenizas y unos carbones fechados en $1745 \pm 20$ años AP [322-391 cal. d.C. $(p=0,58), 252-300$ cal. d.C. $(p=0,37)$ y 399-411 cal. d.C. $(p=0,05)$, calibrado a 2 sigmas, Código de laboratorio: GifA\#17282ECHo\#1713], es decir, es contemporánea con el sondeo 2. 


\section{Modos de asentamiento y producción: una visión desde la tecnología}

\section{Arquitectura: un análisis preliminar}

Hasta el presente, el sitio Antigal Motaite es la única aldea temprana conocida en el valle de Barrancas. Tiene una extensión de ca. $45.000 \mathrm{~m} 2$ y está compuesta por 37 estructuras de formas circulares $(n=14)$, semi-circulares $(n=6)$, rectangulares $(n=3)$, rectangulares abiertas $(n=3)$, muros de contención $(n=7)$ y muros indeterminados y/o discontinuos $(n=4)$ (Figura 2). Se aclara que 37 constituye un número mínimo de estructuras. En efecto, los procesos erosivos exponen nuevos recintos de un año para el otro. Asimismo, tal como lo revelan los sondeos presentados previamente, se han detectado derrumbes y cimientos de estructuras enterradas.

Las estructuras están conformadas por el apilamiento de bloques de ignimbrita (Figura 3), desprendidos de los farallones localizados al otro lado del río Barrancas y de su afluente al norte del sitio. Los muros se realizaron mediante la técnica de "pirca seca" (sin argamasa); fueron construidos con bloques de diferentes tamaños, seleccionados y acomodados minuciosamente de modo que entre las juntas apenas quedaron mínimos intersticios. La dispersión de bloques en torno a los cimientos, tanto en superficie como en capa, se debería a derrumbes posteriores a la(s) ocupación(es). Debido a la homogeneidad composicional y textural de los sedimentos, sumada a la intensidad de los procesos erosivos, no se ha podido determinar un piso de ocupación o una capa común a los recintos y los muros. En algunos sectores sondeados, sin embargo, se pudieron identificar segmentos de estructuras por debajo del piso actual, posiblemente cimientos de muros colapsados (Figura 3), aunque no se descarta que pudieran corresponder a distintos eventos de construcción.

El promedio y la mediana del largo máximo son particularmente bajos en las estructuras circulares y particularmente altos en los recintos rectangulares (Tabla 1). El coeficiente de variación sigue una tendencia baja en los recintos circulares, pero en los muros de contención es el más alto. Sin embargo, esta variación podría deberse al gran tamaño del recinto rectangular $n^{\circ} 19$. El desvío estándar y la varianza indican que los recintos rectangulares poseen la variabilidad más significativa (Tabla 1). Se puede entonces observar que, en general, las estructuras circulares son más pequeñas y menos variables, mientras que los recintos rectangulares y los muros de contención son más diversificados y, generalmente, mucho más amplios o extensos.

\begin{tabular}{|l|c|c|c|c|c|}
\hline & Circular & Semi-circular & $\begin{array}{c}\text { Rectangular } \\
\text { abierta }\end{array}$ & $\begin{array}{c}\text { Muro de } \\
\text { contención }\end{array}$ & Rectangular \\
\hline $\mathrm{N}$ & 14 & 6 & 4 & 7 & 3 \\
Promedio (m) & 2,9 & 5,3 & 6,2 & 6,5 & 14,3 \\
Varianza & 0,422241 & 6,823627 & 2,775833 & 23,9918 & 45,44083 \\
Desvío estándar & 0,649801 & 2,612207 & 1,666083 & 4,898142 & 6,740982 \\
Mediana (m) & 2,9 & 5,5 & 6,2 & 5 & 16 \\
Coef. Var & 22,53524 & 49,64184 & 26,9811 & 75,77236 & 47,19474 \\
\hline
\end{tabular}

Tabla 1. Estadísticas univariadas del largo máximo de las estructuras (m).

Finalmente, se pueden diferenciar tres grandes sectores separados espacialmente siguiendo un eje sudoeste-nordeste. Al sur, un recinto rectangular parece estar relacionado con muros de contención. En el sector intermedio, algunos recintos 


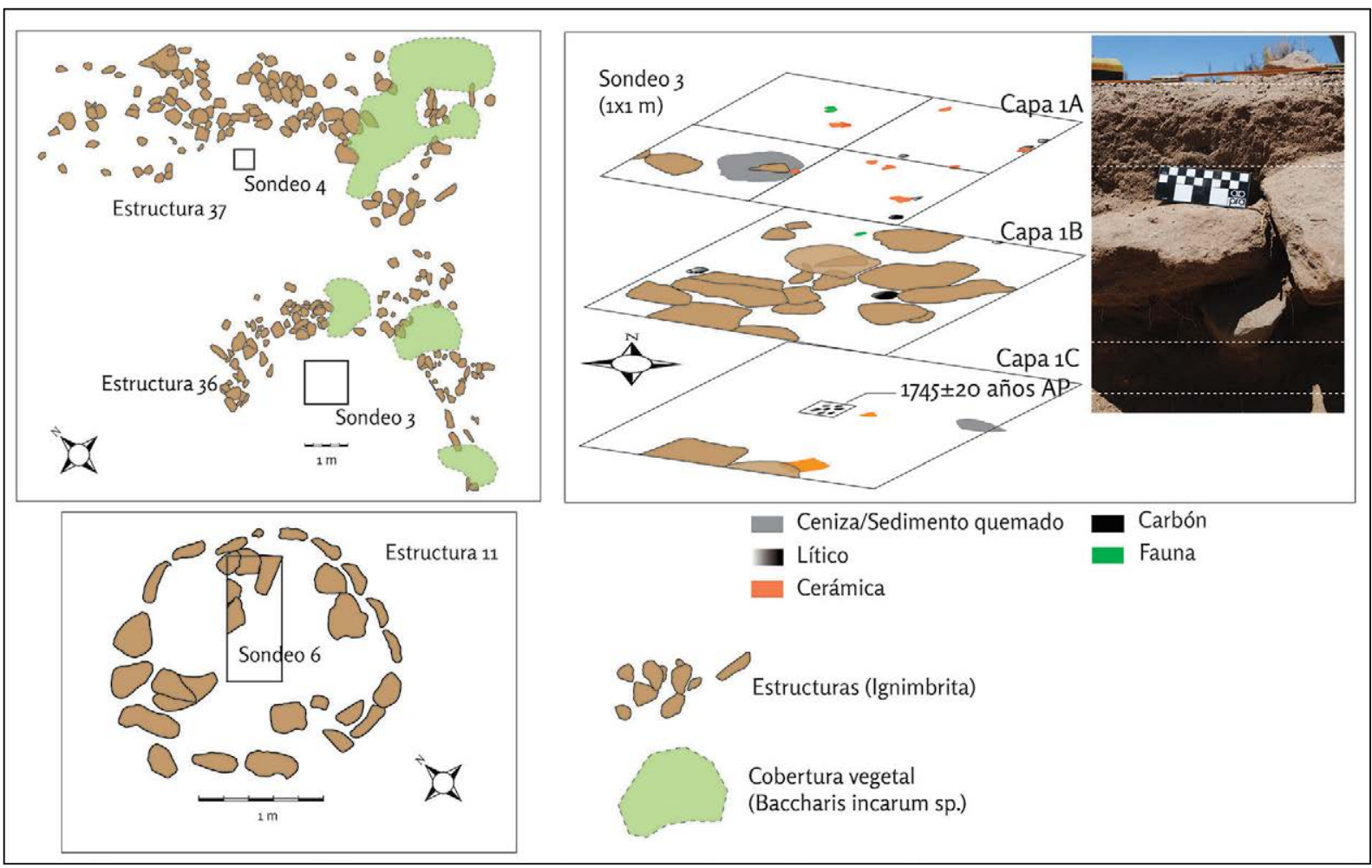

Figura 3. Representación de algunas estructuras de Antigal Motaite. Ubicación de los sondeos relacionados y detalle de la estratigrafía del sondeo 3.

circulares y semi-circulares se vinculan directamente entre sí o mediante el contacto con alguna pared. Distintos muros de contención están también conectados entre sí. En el último sector, hacia el norte del sitio, se destacan dos estructuras rectangulares de grandes dimensiones en relación con los demás recintos, presentando una pared en común. En este sector, varios recintos circulares, semi-circulares y muros de contención también están conectados (Figura 2).

\section{Tecnología lítica: un análisis desde las cadenas operativas}

En una primera etapa de análisis, es pertinente mencionar la ubicación de las fuentes de las materias primas representadas en el registro arqueológico. La cuarcita es una materia prima que abunda en la cuenca y es altamente disponible, aunque su calidad para la talla es variable. Diversas rocas silíceas y ópalos también están disponibles localmente, bajo la forma de nódulos pequeños $(<10 \mathrm{~cm})$ y con superficies muy irregulares, situación que significaría una restricción según varias técnicas de talla. En cuanto a la andesita, no es inmediatamente local. Hay afloramientos de esta roca ca. $20 \mathrm{~km}$ al norte del sitio, pertenecientes al Complejo Ígneo Santa Ana compuesto por diques básicos y lavas andesítico-dacíticas (Seggiaro, Becchio, Bercheñi y Ramallo, 2015). Del mismo modo, no se han registrado afloramientos de obsidiana en la cuenca. El más cercano sería el de la Caldera Vilama a ca. $80 \mathrm{~km}$ de distancia; los otros afloramientos conocidos como Zapaleri-Laguna Blanca, Tocomar y Quirón, entre otros, están a más de 100 km lineales de distancia. En la mayoría de los afloramientos mencionados, los nódulos no superan los 10-12 cm; un caso excepcional sería la fuente Zapaleri-Laguna Blanca con nódulos de hasta $20 \mathrm{~cm}$ (Yacobaccio, Escola, Pereyra, Lazzari y Glascock, 2004).

En cuanto a las materias primas representadas en el conjunto lítico del sitio, sobre una muestra total de 446 artefactos, domina la obsidiana $(n=176 ; 39,5 \%)$, seguida por la 
cuarcita $(n=170 ; 38,1 \%)$. Para la primera, las etapas de confección de instrumentos están bien representadas $(n=137)$. Los instrumentos formatizados $(n=12)$ dominan sobre los instrumentos retocados $(n=6)$. Al contrario, en el caso de la cuarcita, las lascas de desbaste $(n=94)$ y los instrumentos retocados $(n=22)$ están en mayor representación. Esta situación lleva a plantear que estas dos materias primas habrían sido objeto de estrategias de producción distintas. Las otras materias primas, la andesita $(n=37 ; 8,3 \%)$ y el ópalo $(n=43 ; 9,6 \%)$, están particularmente representadas a través de lascas de confección. En muy bajas proporciones, se puede mencionar la presencia de cuarzo $(n=11 ; 2,5 \%)$ y de una variedad de cuarcita de grano muy fino ( $n$ $=9 ; 2 \%$. En general, se observa que los instrumentos retocados $(n=37 ; 8,3 \%)$ dominan sobre los instrumentos formatizados $(n=29 ; 6,5 \%)$ (excepto en el caso de la obsidiana), hecho que refleja que las UTF fueron buscadas y obtenidas desde la producción de formas base que no requerían formatización, sino solamente un retoque.

En particular para la obsidiana, los estudios FRX muestran la amplia dominancia de Zapaleri-Laguna Blanca sobre toda otra fuente (Tabla 2). Es llamativo que no se hubiera detectado Caldera Vilama, fuente también ubicada en el norte de la cuenca y más cercana que Zapaleri-Laguna Blanca. Esta procedencia casi exclusiva, sumada al hecho de que la obsidiana es la materia prima dominante y cuyos instrumentos resultaron más de las actividades de confección que de desbaste, refleja una característica particular de esta roca en el sitio, lo cual es consecuencia de estrategias de aprovisionamiento y de producción totalmente distintas de las registradas para la cuarcita.

\begin{tabular}{|c|c|c|c|c|c|c|c|c|c|c|}
\hline $\mathbf{N}^{\circ}$ & $\mathbf{M n}$ & $\mathrm{Fe}$ & $\mathrm{Zn}$ & $\mathbf{R b}$ & $\mathrm{Sr}$ & $Y$ & $\mathrm{Zr}$ & $\mathrm{Nb}$ & Th & $\begin{array}{c}\text { Fuente } \\
\text { asignada }\end{array}$ \\
\hline 101 & 392 & 7891 & 44,4 & 203,9 & 132,0 & 34,5 & 211,2 & 22,5 & 23,2 & Zap.-LB \\
\hline 102 & 540 & 12151 & 63,9 & 230,1 & 146,0 & 38,1 & 201,2 & 26,4 & 23,9 & Zap.-LB \\
\hline 103 & 476 & 9527 & 50,5 & 211,4 & 131,6 & 34,4 & 190,7 & 22,7 & 22,0 & Zap.-LB \\
\hline 104 & 516 & 14849 & 66,3 & 227,1 & 148,6 & 38,3 & 258,8 & 25,0 & 23,6 & Zap.-LB \\
\hline 105 & 497 & 7112 & 23,4 & 541,1 & 16,0 & 23,1 & 30,3 & 84,3 & 0,6 & Quirón \\
\hline 106 & 482 & 10189 & 54,8 & 220,6 & 139,9 & 35,2 & 188,4 & 24,1 & 24,1 & Zap.-LB \\
\hline 107 & 433 & 11381 & 52,4 & 210,6 & 135,8 & 34,5 & 186,8 & 24,1 & 22,6 & Zap.-LB \\
\hline 108 & 446 & 11767 & 56,5 & 220,5 & 140,3 & 35,1 & 194,4 & 23,3 & 22,9 & Zap.-LB \\
\hline 109 & 446 & 9782 & 54,3 & 218,3 & 136,7 & 35,6 & 218,5 & 22,6 & 22,1 & Zap.-LB \\
\hline 110 & 464 & 9403 & 50,2 & 207,8 & 130,5 & 33,6 & 191,6 & 22,2 & 20,9 & Zap.-LB \\
\hline 111 & 464 & 12099 & 58,6 & 225,1 & 143,8 & 35,0 & 211,0 & 23,6 & 20,6 & Zap.-LB \\
\hline 112 & 389 & 11830 & 52,9 & 209,6 & 137,4 & 33,4 & 190,7 & 22,7 & 20,7 & Zap.-LB \\
\hline 113 & 481 & 13089 & 60,7 & 231,0 & 147,7 & 36,3 & 203,9 & 25,8 & 23,9 & Zap.-LB \\
\hline 114 & 435 & 10038 & 48,6 & 207,7 & 131,6 & 33,3 & 189,0 & 23,0 & 23,9 & Zap.-LB \\
\hline 115 & 381 & 8435 & 41,4 & 184,1 & 119,3 & 31,9 & 169,2 & 22,0 & 20,5 & Zap.-LB \\
\hline 116 & 433 & 8906 & 48,4 & 206,7 & 134,0 & 34,9 & 187,4 & 24,5 & 22,1 & Zap.-LB \\
\hline 117 & 406 & 12387 & 53,0 & 211,6 & 137,0 & 35,0 & 204,6 & 22,6 & 21,0 & Zap.-LB \\
\hline 118 & 444 & 9206 & 47,1 & 208,3 & 133,0 & 34,4 & 235,8 & 23,5 & 20,1 & Zap.-LB \\
\hline 119 & 465 & 10263 & 59,0 & 217,7 & 137,9 & 35,1 & 202,5 & 24,3 & 21,8 & Zap.-LB \\
\hline 120 & 450 & 9630 & 49,4 & 199,4 & 126,3 & 32,4 & 181,0 & 21,4 & 19,7 & Zap.-LB \\
\hline 121 & 483 & 10555 & 49,8 & 221,7 & 139,9 & 34,1 & 192,7 & 24,7 & 22,2 & Zap.-LB \\
\hline 122 & 477 & 10262 & 53,9 & 222,0 & 141,5 & 36,1 & 192,7 & 24,2 & 22,8 & Zap.-LB \\
\hline 123 & 435 & 10555 & 54,3 & 219,2 & 140,6 & 34,3 & 208,0 & 23,8 & 22,9 & Zap.-LB \\
\hline 124 & 478 & 11659 & 56,4 & 221,4 & 142,5 & 35,8 & 233,7 & 24,3 & 22,5 & Zap.-LB \\
\hline
\end{tabular}

Tabla 2. Concentración de elementos (ppm) en artefactos de obsidiana medidos por Fluorescencia de Rayos X (FRX). 
En general, se puede observar cierta diversidad en los métodos de desbaste, pero con un dominio del Tipo $C$ (Figura 4). Los tipos $C$ presentan una variabilidad característica de su flexibilidad inherente, y del saber-hacer de los talladores para buscar la forma base deseada, pero con la desventaja de una producción limitada. Se observa la presencia del desbaste laminar, aunque con una escasa presencia de sus formas base (hojas) descartadas en el sitio. Esta forma de desbaste está representada solamente por los núcleos. Al contrario, el desbaste centrípeto/discoidal está más visible a través de las formas base y de los instrumentos que de los núcleos. Los métodos SASP y otros tipos $D$ tienen cierta representatividad, particularmente entre los núcleos (Figura 4). Este hecho podría ser el resultado de la equifinalidad entre las lascas obtenidas durante las primeras etapas de aplicación de esos métodos y las producidas mediante un desbaste de Tipo $\mathrm{C}$, por lo cual se podría contemplar una sobreestimación de este último.

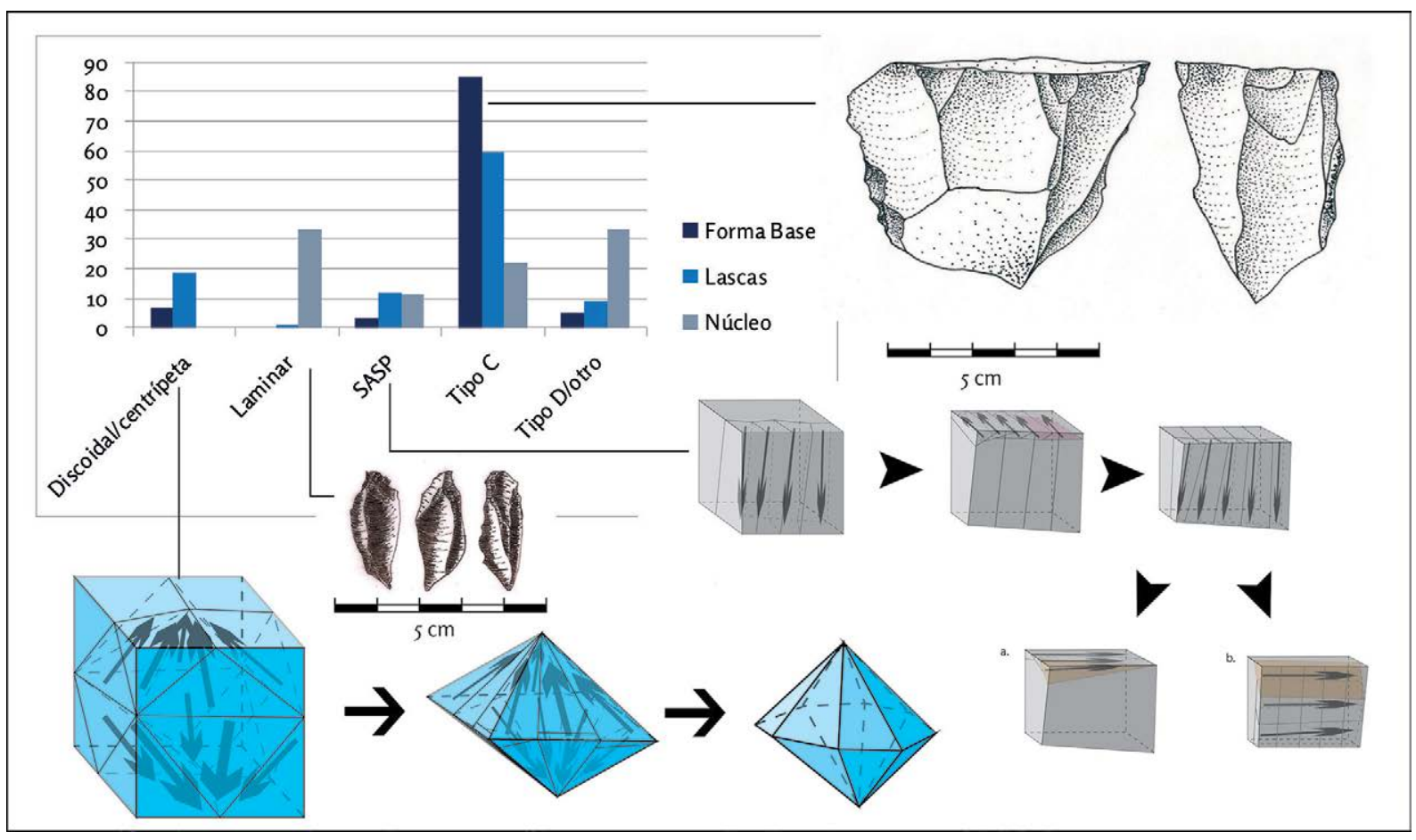

Figura 4. Representación de los métodos de desbaste en relación con sus distintos tipos de productos (Núcleos, Lascas y Formas base de instrumentos).

Entre los instrumentos $(n=81)$, se distinguen las puntas de proyectil (Figura 5). Entre ellas, se destaca el tipo de limbo triangular con aletas y pedúnculo (Figura 6), característico de los contextos tempranos puneños (Aschero y Hocsman, 2011; Escola, 2002; entre otros). En su mayoría están confeccionadas en obsidiana, pero también, aunque en menor medida, en ópalo y cuarcita. La producción de la forma base es indiferenciada (posiblemente de tipo C), dado que, cuando pudo ser identificada, el eje técnico siempre fue distinto del eje morfológico. Además, se pudieron observar diferentes esquemas de confección y diversidad morfológica, lo cual concordaría con una adecuación del saber-hacer de los talladores sobre una variabilidad de formas base. Estas puntas fueron confeccionadas por presión, tal como lo muestran las extracciones paralelas invasivas de arista tenue. Algunas son unifaciales y otras son bifaciales, pero con tratamiento jerárquico de las superficies (Boëda, 2013) siendo, en este caso, de sección plano-convexa. 


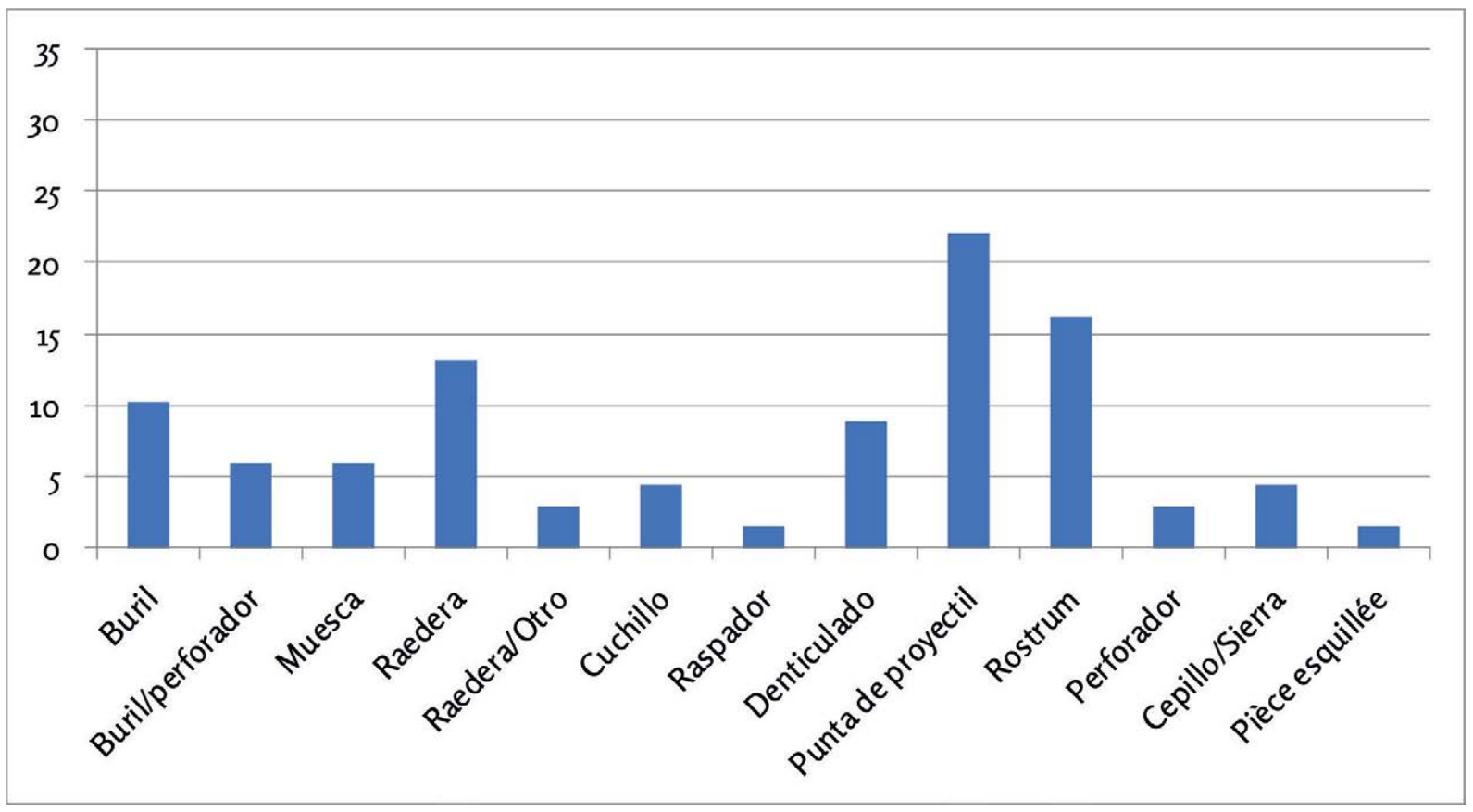

Figura 5. Porcentajes de los instrumentos en el sitio.

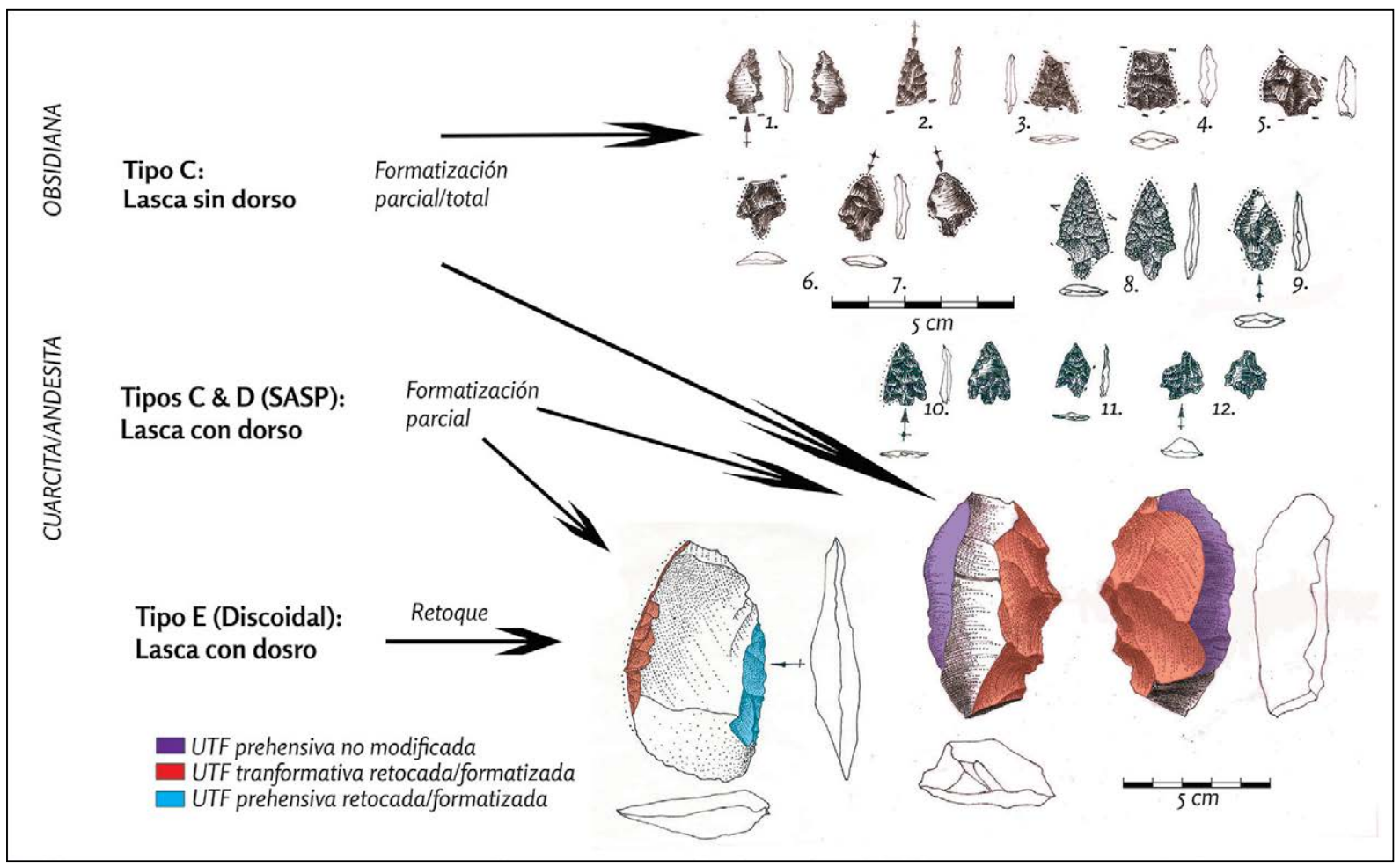

Figura 6. Trayectorias tecnológicas de los instrumentos. 
Asimismo, se registraron "rostrii" (ver Hoguin y Kohan, 2018; Kohan, 2018), raederas y buriles. Estos instrumentos están confeccionados sobre lascas centrípetas, con dorso, y a su vez, sobre nódulos. Se ha notado también la presencia de raederas y de buriles en proporciones mayores a 10\%. Sin embargo, en general, se destaca una distribución diversificada cuyas categorías están en bajas proporciones, $<10 \%$ (Figura 5).

En cuanto a la prehensión de los instrumentos, determinados por las UTF prensiles, se destaca que la prehensión manual representa el $75 \%(n=48)$ de los instrumentos donde se pudieron identificar tales partes, lo cual es ampliamente mayor al porcentaje del sitio Alero Morro Blanco (a unos $6 \mathrm{~km}$ al norte de Antigal Motaite) que ya se diferenciaba por esta clase de prehensión durante el Holoceno medio (Hoguin y Kohan, 2018). Entre las UTF prensiles, se destacan particularmente los dorsos obtenidos desde el desbaste (y no desde la formatización), una característica particular de este sitio, representadas en un $57 \%(n=36)$ de los instrumentos. Por otro lado, los pedúnculos (fuertemente relacionados con las puntas de proyectil y la obsidiana) están relativamente bien representados $(22 \% ; n=14)$ y se vinculan con la formatización posterior al desbaste.

En términos generales, se pueden esbozar dos tipos de cadenas operativas: las de cuarcita, para instrumentos de procesamiento (principalmente rostrii, raederas y buriles) con formas base con dorso obtenidas mediante distintos métodos de desbaste (Tipo C, SASP, centrípeto/discoidal), luego retocados marginalmente, y las de obsidiana, principalmente dedicadas a la manufactura de cabezales a partir de la obtención de pequeñas lascas, luego formatizadas por presión (Figura 6).

\section{Tecnología cerámica: un análisis con énfasis en la composición de las pastas}

La cerámica de Antigal Motaite (AM) es representativa de una alfarería de tipo ordinaria o utilitaria. Si bien es mayormente lisa y monocroma, se recuperaron también escasos tiestos decorados, pintados y grabados. En algunos casos, el nivel tecnológico y la composición de las pastas sugieren que, al menos, una parte pudo haber sido manufacturada en otros lugares, aunque no fue posible corroborar aún si se trata de regiones alejadas o de zonas que, estando fuera de los límites de la cuenca investigada, podrían estar comprendidas dentro del radio de acción de los ocupantes del sitio. La metodología se focalizó en la determinación de los antiplásticos. Este análisis permite establecer correlaciones, en principio con las formaciones geológicas locales. El análisis de las pastas sugiere que la mayoría de las litologías observadas en las cerámicas del sitio se corresponderían con unidades formacionales de importante desarrollo local que, a su vez, se extienden ampliamente en el ámbito de la Puna jujeño-salteña.

Una característica destacable en esta cerámica es la recurrencia del mismo tipo de antiplásticos, aun tratándose de composiciones abigarradas. Generalmente, la carga (antiplásticos) de estas pastas contiene gránulos o microfragmentos de sedimentitas pelitas y areniscas - y metasedimentitas -lutitas y pizarras-, estas últimas dominantes en la mayoría de las muestras. Las litologías mencionadas son parte de las formaciones ordovícicas que en el área constituyen la Serranía de Coyahuaina (Seggiaro et al., 2015; Zappettini, 1989). Asimismo, los productos de su descomposición física están representados en las arenas, las gravas y los cantos rodados de la cuenca.

Esta alfarería es mayoritariamente rojiza, abarcando variadas gamas dentro de las tonalidades rojo ladrillo, marrón rojizo y gris rojizo (Tabla 3). Debido al grado de

1 Instrumento de filo convexo que presenta una protuberancia, más estrecha que en un raspador y más ancha que en un buril. 
fragmentación, son escasos los tiestos reconocidos como derivados de una misma pieza, es decir que el número mínimo de piezas estimado (NMP $=105)$ prácticamente coincide con el número de tiestos recuperados $(n=111)$. Los tratamientos superficiales incluyen alisado burdo (peinado), alisado, engobe y falso engobe, engobe con agregado de pigmento rojo y pulido. Los fragmentos de cerámica decorada son exiguos $(n=5)$ y corresponden a cuatro fragmentos pintados (Figura 7 A, B y C), uno de ellos con decoración pintada en negativo -rojo/amarillo rojizo- (Figura 7 C) y el cuarto es un borde de vasija pequeña (¿puco?) que tiene una línea negra sobre la mitad interna del labio plano (Figura $7 \mathrm{~L}, \mathrm{AM} 11$ ). El restante es un fragmento de ollita (restringida) con decoración grabada en el hombro que consiste en líneas finas que definen motivos cuadrangulares irregulares (Figura 7D). Asimismo, se destacan 3 fragmentos de tubo o caño de pipa de paredes gruesas (10 a $18 \mathrm{~mm}$ ) y diámetros que rondan los $30 \mathrm{~mm}$ (Figura 7 F, G y H).

Se estima que el tamaño de las piezas fue variado. Ha sido dificultoso establecer formas y tamaños de vasijas, aunque se han reconocido vasijas pequeñas y medianas,

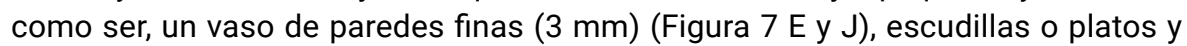
también grandes contenedores, como ollas y tinajas de paredes gruesas (entre $10 \mathrm{y}$ $12,5 \mathrm{~mm}$ de espesor). Un asa recuperada en la E17 (capa 1) se correspondería con una vasija de porte considerable. La mayor parte de los bordes son directos, rectos $\mathrm{y} / \mathrm{o}$ ligeramente evertidos en vasijas de formas simples, generalmente no restringidas (Figura $7 \mathrm{I}$ a N). Se sostiene que las piezas fueron levantadas mediante la técnica de adición de rollos o enrollamiento, excepto la técnica de manufactura de las pipas, que no ha sido determinada aún.

Las litologías de los antiplásticos permitieron definir los tipos de pasta en esta muestra cerámica. La clasificación se basó en la presencia de componentes policristalinos -fragmentos de sedimentitas y metasedimentitas - y de minerales como cuarzo, feldespatos (s.l.) y micas. Dicha clasificación fue establecida mediante el análisis bajo estéreo microscopio de los 111 tiestos recolectados en el sitio. Las pastas se agruparon en 4 clases según la composición y la abundancia relativa de los antiplásticos²:

1. metasedimentita gris $y / o$ rojiza $(34 \%)$

2. metasedimentita gris y/o rojiza (mayoritaria) + cuarzo/cuarcita [con/sin feldespatos (s.I.)] (33\%)

3. metasedimentita gris y/o rojiza + lutitas/micacitas blancas + cuarzo/cuarcita $(19 \%)$

4. metasedimentita gris y/o rojiza y/o cuarzo/cuarcita (mayoritarios) + mica + otros minerales $\mathrm{n} / \mathrm{d}$ (en proporciones muy minoritarias) (14\%)

En síntesis, las muestras estudiadas bajo microscopio petrográfico son compatibles con los tipos 2 (AM1 y AM3), 3 (AM4, AM5, AM6 y AM7) y 4 (AM2, AM8 y AM9) definidos previamente (Ver arriba). El tipo 1 no se ha visto representado en la cerámica analizada con microscopio de polarización (su ausencia podría deberse a las diferentes escalas de observación y al instrumental utilizado). La muestra AM9 podría corresponderse con el estilo cerámico policromo denominado Vaquerías (Bugliani y Domingorena, 2012). Este tiesto, único en su estilo en $A M$, está siendo analizado con mayor detalle.

En líneas generales, la cerámica de Antigal Motaite tiene pastas heterogéneas en composición y textura, con partículas fracción arena muy fina a grava fina. Si bien algunas de las cerámicas analizadas podrían ser alóctonas, la presencia recurrente 


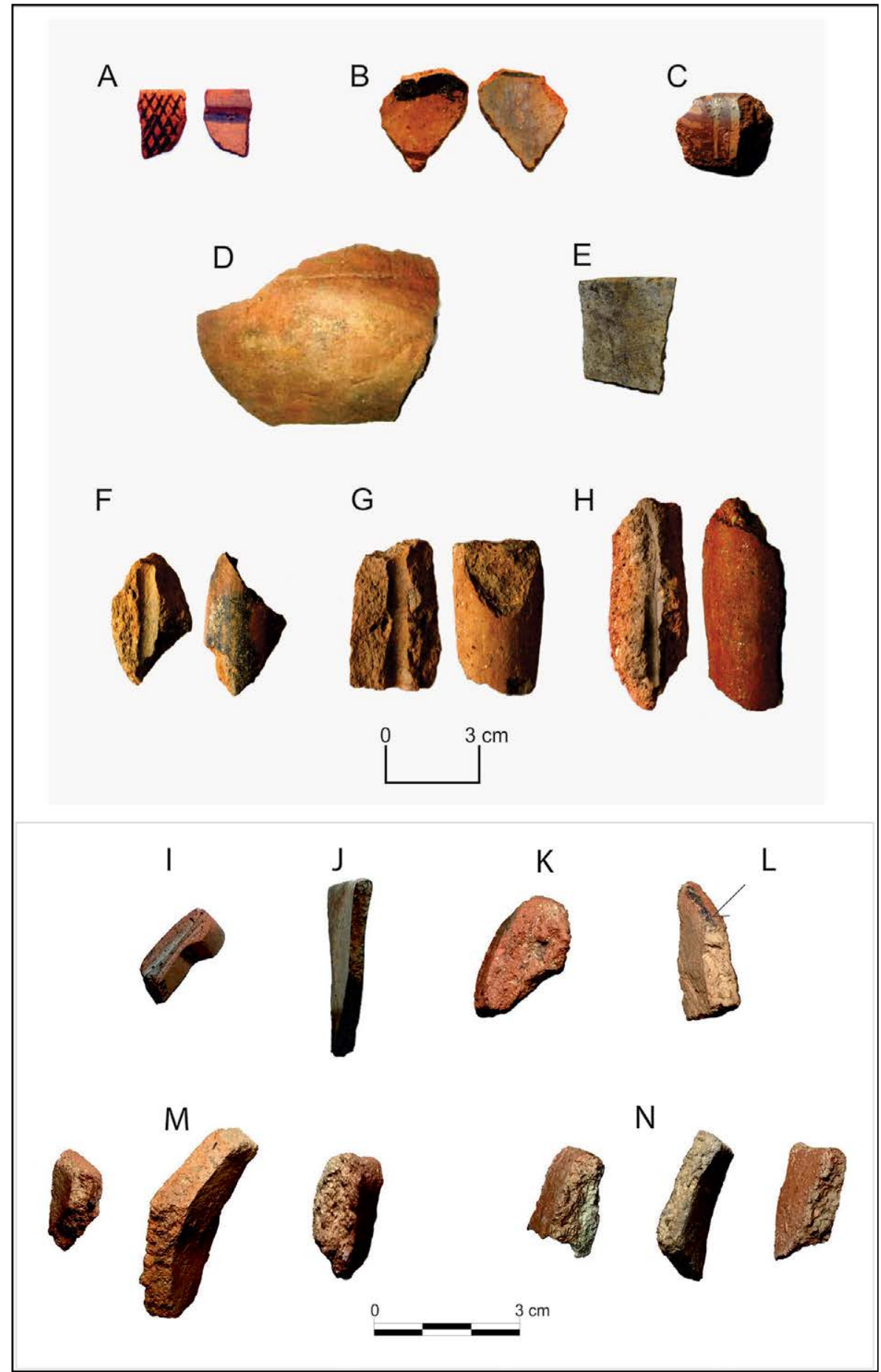

Figura 7. Cerámica de Antigal Motaite. A) AM1;B) AM8; C) AM9 (tipo ¿Vaquerías?) D) AM10; E) AM7; F, G y H) respectivamente $A M 5,4$ y 6 . Diferentes tipos de bordes: I) AM1; J) AM7; K) AM9; L) AM11; $M$ y N) Otros bordes.

de fragmentos de litologías locales en las pastas, por ejemplo, las metasedimentitas grises y/o rojizas, nos hace reflexionar sobre el origen de estas materias primas. La abundancia de dichas metasedimentitas, conjuntamente con cuarzo y feldespatos (s.l.), podría estar indicando un origen local para la mayoría de las pastas analizadas (Ver más arriba y Figura 8). Sin embargo, las composiciones observadas también suelen encontrarse en pastas cerámicas registradas en sitios tardíos que se encuentran al 


\begin{tabular}{|c|c|c|c|c|c|c|c|}
\hline Muestra & Procedencia & $\begin{array}{c}\text { Tipo de } \\
\text { fragmento y } \\
\text { forma de vasija }\end{array}$ & $\begin{array}{l}\text { Tratamiento } \\
\text { superficial }\end{array}$ & $\begin{array}{c}\text { Color sup. I } \\
\text { (Munsell) }\end{array}$ & $\begin{array}{c}\text { Color sup. E } \\
\text { (Munsell) }\end{array}$ & $\begin{array}{l}\text { Decoración } \\
\text { (sup. I) }\end{array}$ & $\begin{array}{l}\text { Decoración } \\
\text { (sup. E) }\end{array}$ \\
\hline $\mathrm{AM} 1^{(*)}$ & Sup (T2) & $\begin{array}{l}\text { borde reforzado } \\
\text { de plato o } \\
\text { escudilla }\end{array}$ & engobe (I y E) & rojo $(2,5 Y R 5 / 6)$ & $\begin{array}{c}\text { marrón } \\
\text { rojizo claro } \\
(2,5 Y R 6 / 4)\end{array}$ & $\begin{array}{l}\text { pintura negra } \\
\text { (reticulado fino) }\end{array}$ & $\begin{array}{c}\text { pintura negra } \\
\text { (banda paralela } \\
\text { al borde) }\end{array}$ \\
\hline $\mathrm{AM} 2^{(*)}$ & Sup (T2) & $\begin{array}{l}\text { cuerpo (vasija } \\
\text { de forma } n / d \text { ) }\end{array}$ & alisado (I y E) & rojo $(10 R 5,5 / 5)$ & $\begin{array}{c}\text { rojo } \\
(10 \mathrm{R} 5,5 / 3,5)\end{array}$ & - & - \\
\hline $\mathrm{AM}^{(*)}$ & Sup (T2) & $\begin{array}{l}\text { cuerpo (vasija } \\
\text { de forma } n / d \text { ) }\end{array}$ & engobe (I y $E$ ) & $\begin{array}{l}\text { rojo claro } \\
(10 \mathrm{R} 5 / 4)\end{array}$ & $\begin{array}{l}\text { rojo claro } \\
\text { (10R5/2) }\end{array}$ & - & - \\
\hline $\mathrm{AM} 4^{(*)}$ & Sup (T5) & caño de pipa & $\begin{array}{l}\text { falso engobe } \\
\text { pulido }(E)\end{array}$ & $\begin{array}{c}\text { rojo claro } \\
(7,5 \mathrm{R} 3,5 / 2)\end{array}$ & $\begin{array}{c}\text { marrón rojizo } \\
\text { claro } \\
(5 Y R 6 / 4)\end{array}$ & - & - \\
\hline $\mathrm{AM}^{(*)}$ & Superficie & caño de pipa & $\begin{array}{l}\text { engobe pulido } \\
\text { (E) }\end{array}$ & $\begin{array}{l}\text { rojo claro } \\
(7,5 \mathrm{R} 5 / 3)\end{array}$ & $\begin{array}{c}\text { gris osc/rojo } \\
\text { claro }(7,5 \mathrm{R} 3,5 / 1 \\
\text { a } 7,5 \mathrm{R} 5 / 2)\end{array}$ & - & - \\
\hline $\mathrm{AM} 6^{(*)}$ & Sup (GPS) & caño de pipa & $\begin{array}{l}\text { falso engobe } \\
\text { pulido }(\mathrm{E})\end{array}$ & $\begin{array}{c}\text { gris/rojo claro } \\
(2,5 Y 6 / 1 \mathrm{a} \\
\text { 2,5YR6/6) }\end{array}$ & $\begin{array}{l}\text { rojo claro } \\
(2,5 \mathrm{YR6} / 4)\end{array}$ & - & - \\
\hline $\mathrm{AM}^{(*)}$ & Superficie & borde de vaso & $\begin{array}{l}\text { falso engobe } \\
(E)\end{array}$ & $\begin{array}{l}\text { gris rosado } \\
(7,5 Y \mathrm{YR} 6 / 2)\end{array}$ & $\begin{array}{c}\text { gris } \\
(7,5 Y R 5,5 / 1)\end{array}$ & - & - \\
\hline $\mathrm{AM} 8^{(*)}$ & Superficie & $\begin{array}{l}\text { cuerpo (vasija } \\
\text { de forma } n / d)\end{array}$ & Engobe (I y E) & rojo $(2,5 Y R 5 / 6)$ & $\begin{array}{l}\text { gris rojizo } \\
\text { (5YR5/2) }\end{array}$ & $\begin{array}{c}\text { pintura negra y } \\
\text { marrón (bandas } \\
\text { subparalelas) }\end{array}$ & $\begin{array}{c}\text { pintura negra } \\
\text { (banda paralela } \\
\text { a la banda } \\
\text { negra I) }\end{array}$ \\
\hline AM9 & Superficie & $\begin{array}{l}\text { borde (vasija de } \\
\text { forma } n / d)\end{array}$ & $\begin{array}{l}\text { engobe pulido } \\
\text { (I y } E)\end{array}$ & $\begin{array}{c}\text { amarillo rojizo } \\
(5 Y R 6 / 6)\end{array}$ & $\begin{array}{c}\text { pintura roja } \\
(5 \mathrm{R} 4 / 3)\end{array}$ & $\begin{array}{l}\text { pintura roja } \\
\text { (5R4/6) (en } \\
\text { negativo) }\end{array}$ & $\begin{array}{c}\text { pintura roja } \\
\quad(5 \mathrm{R} 4 / 6)\end{array}$ \\
\hline AM10 & $\begin{array}{l}\text { E17-S3 } \\
\text { extensión - } \\
\text { capa 1a }\end{array}$ & cuerpo de ollita & alisado & $\begin{array}{c}\text { rojo claro } \\
(10 \mathrm{R} 6 / 4)\end{array}$ & rojo (10R5/3) & - & $\begin{array}{l}\text { grabada (líneas } \\
\text { rectas) }\end{array}$ \\
\hline AM11 & Sup (GPS) & $\begin{array}{l}\text { borde (vasija de } \\
\text { forma } n / d)\end{array}$ & $\begin{array}{l}\text { alisado burdo } \\
\text { ("peinado") }\end{array}$ & $\begin{array}{c}\text { marrón rojizo } \\
\text { claro } \\
(2,5 \mathrm{YR} 6 / 4)\end{array}$ & Rojo (10R5/6) & $\begin{array}{l}\text { pintura negra } \\
\text { (sobre labio } \\
\text { plano, mitad } \\
\text { interna) }\end{array}$ & - \\
\hline
\end{tabular}

Tabla 3. Caracterización de la cerámica decorada y de los tiestos analizados microscópicamente. Referencias: Sup (superficie), I (interna), E (externa), $n / d$ (no determinada), GPS (punto georreferenciado), (*) Muestra analizada microscópicamente.

norte, oeste y sur de la cuenca de Barrancas. Estas coincidencias composicionales estarían relacionadas con la gran extensión que presentan los afloramientos ordovícicos en la Puna. En la cuenca del río Barrancas, estas unidades constituyen la Serranía de Coyahuaina de rumbo general nor-noreste. En el sur, la prolongación meridional de esta serranía es la Sierra de los Cobres, en cuya ladera oriental además afloran el basamento precámbrico y las secuencias cámbricas; hacia el norte, las unidades ordovícicas son parte del sustrato puneño (cubierto por formaciones terciarias y cuaternarias), aflorando nuevamente en las Sierras de Cochinoca y Escaya y extendiéndose más allá de la frontera con Bolivia. Al mismo tiempo, la presencia en las pastas de fragmentos de arcillitas, probablemente transformadas en micacitas blancas durante el proceso de cocción de la cerámica, sugiere fuentes que podrían encontrarse en cuencas alejadas, como por ej., las arcillas caoliníticas blanco-amarillentas de la Fm. Tafna (Neógeno) que aflora en la región de Casira, Calahoyo y Tafna, localidades ubicadas a escasa distancia de la frontera argentino-boliviana (Rubiolo et al., 2003).

\section{Discusión y conclusiones}

El asentamiento de Antigal Motaite está compuesto por un mínimo de 37 estructuras y pudo haber albergado un grupo relativamente extendido de personas, reunidas tanto estacionalmente como de forma más permanente. A la luz de las distintas líneas de 


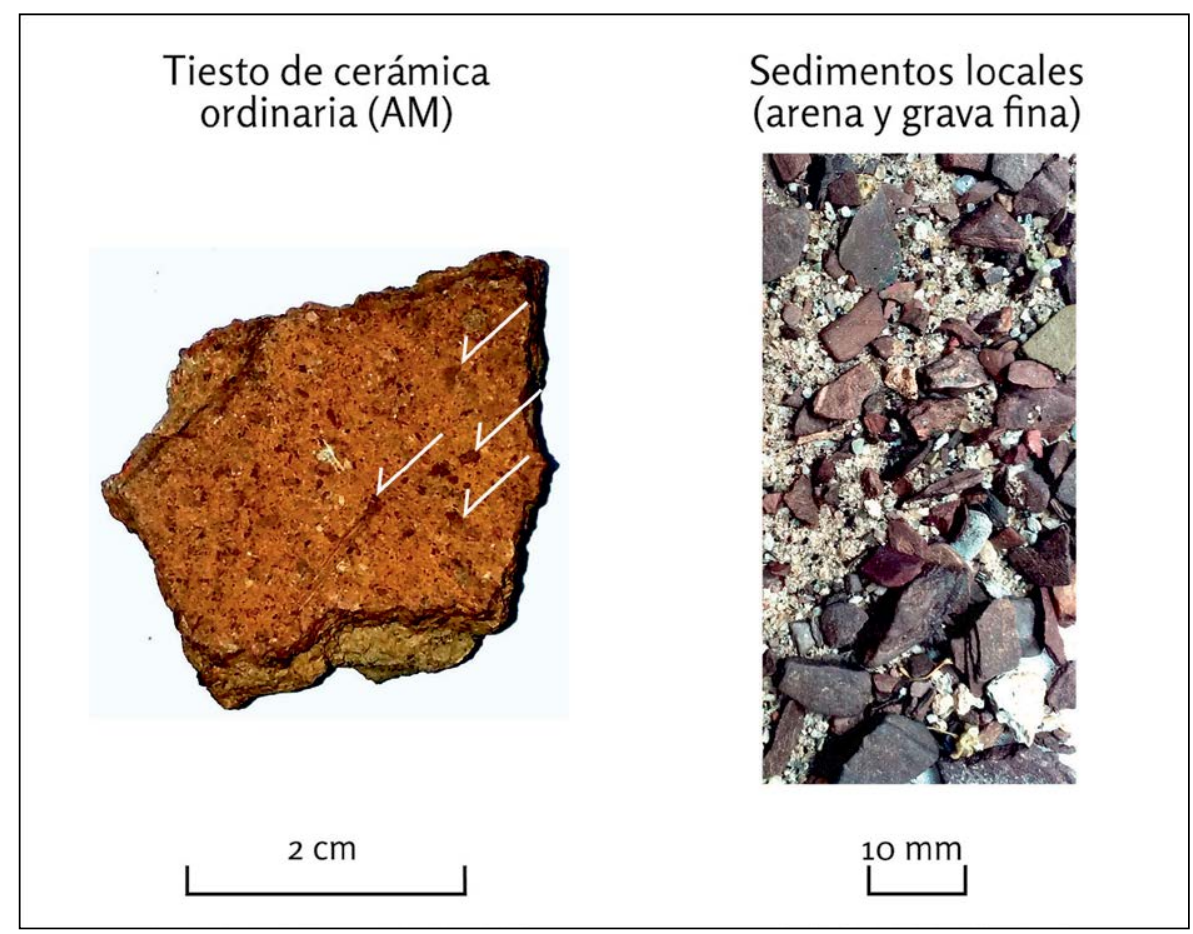

Figura 8. Cerámica ordinaria de AM (las flechas señalan algunos de los fragmentos de metasedimentitas gris/ rojizas). A la derecha, los sedimentos locales muestran las mismas litologías.

evidencia presentadas y en comparación con los antecedentes regionales, se propone que el sitio fue una base residencial. A continuación, se evalúa la pertinencia de las distintas hipótesis presentadas en este marco.

La presencia de algunos recintos rectangulares de mayor extensión se relacionaría con espacios comunales. Los pequeños montículos circulares cerrados podrían ser interpretados como estructuras de almacenamiento puesto que, por su superficie, se descarta que hubiesen sido habitacionales. La función residencial habría sido cumplida por los recintos semi-circulares de mayor tamaño, aptos para albergar una familia nuclear. Se puede plantear que posiblemente no todas las estructuras fuesen coetáneas, ya que los recintos circulares suelen ser más antiguos que los rectangulares, como es el caso de los sitios de Capinte Arriba y Pueblo Viejo de Tucute (Albeck, 2010; Albeck y Zaburlín, 2008).

Una hipótesis alternativa podría contemplar los grandes recintos rectangulares (o alguno de ellos) como espacios relacionados con el cautiverio de camélidos domésticos. Cabe recordar que regionalmente la agricultura comienza a tener un impacto importante en las sociedades del Período Temprano, en particular sobre el pastoreo, debido a que el forraje permitiría la permanencia anual de los rebaños en una localidad y, por ende, la de las poblaciones (Grant y Olivera, 2016). En cuanto a las estructuras compuestas por una o varias paredes transversales (no cerradas), y los muros de contención, estarían relacionadas con la jerarquización del espacio agrícola o protección de zonas de cultivo, tal como ha sido documentado en los sitios de Casabindo en los momentos más tempranos de la ocupación en esa área (Albeck, 2010). Estos espacios relativamente reducidos indicarían una agricultura de baja escala y a secano, puesto que no se han relevado canales de riego. Además, el sitio se encuentra levemente sobreelevado con respecto al lecho del río. Estimamos que durante la ocupación del sitio, el río debía estar activo y presentaba buen caudal, como parece corresponderse con la información paleoambiental local que establece condiciones húmedas con ocurrencia 
de eventos torrenciales en el período aquí considerado (Morales et al., 2018; Pirola et al., 2018). Sin embargo, estas hipótesis sobre la funcionalidad de las estructuras requieren una contrastación más robusta a partir de, por ejemplo, estudios químicos y sedimentológicos de suelos, para identificar compuestos orgánicos y/o fitolitos.

Los análisis de la tecnología lítica demuestran la presencia de dos grandes trayectorias en las cadenas operativas: una orientada a la producción por presión de puntas de proyectil enastiladas a partir de un aprovisionamiento no local de obsidianas, y otra dedicada al desbaste de formas base en cuarcita local para confeccionar instrumentos de procesamiento de prehensión manual. A su vez, esta última puede subdividirse en dos itinerarios: (1) producción de lascas indiferenciadas con formatización invasiva, (2) producción de lascas diferenciadas y predeterminadas con retoque marginal o formatización no invasiva. Algunas lascas pueden cumplir con los criterios tecnofuncionales buscados y no requieren mayor formatización. Es relevante hacer notar que uno de estos criterios se basa en la obtención de dorsos, típicos de las lascas flanco de núcleo y discoidales, para la prehensión manual. Así, la producción dominante del sitio en formas base es de tipo $C$, lo cual implica un rendimiento menor de formas base por núcleo pero permite mayor flexibilidad. Por otro lado, una producción menor, aunque presente, de lascas discoidales permitiría una mayor obtención de soportes con criterios tecno-funcionales prefijados (e.g. dorsos), por lo cual serían menos formatizados posteriormente. A su vez, los métodos SASP y otros de tipo D podrían tener un papel intermedio en estas trayectorias, produciendo formas base iguales a las de tipo C y otras más específicas como las lascas flanco de núcleo.

Finalmente cabe destacar que, en el marco de las estrategias de aprovisionamiento de obsidianas, es notable la preferencia de la fuente Zapaleri-Laguna Blanca. Esta observación confirma lo planteado en trabajos anteriores sobre la influencia dominante de la esfera de interacción para la distribución de obsidiana procedente de Zapaleri-Laguna Blanca a largas distancias desde ca. 2000 años AP (Yacobaccio et al., 2004). La obsidiana pudo haber sido uno de los bienes que circularon mediante el tráfico caravanero (Yacobaccio, 2012), y según fuera planteado en trabajos anteriores, éste tuvo una influencia notable sobre el cambio tecnológico (Hoguin y Kohan, 2018; Yacobaccio et al., 2020). Los resultados de este trabajo ponen de manifiesto una cadena operativa de obsidiana y un sistema técnico de armas que funcionan de manera autónoma con respecto al resto de la producción lítica. En efecto, la producción de puntas de proyectil en obsidiana requiere de un sistema de arco y de un enastilamiento para volverse funcional. Todo hace pensar que la obsidiana del sitio no fue una roca complementaria, sino que se usó exclusivamente en el marco de este sistema técnico. Por el contrario, las actividades de procesamiento llevaron a la producción de instrumentos preferencialmente de cuarcita, no enmangados, sino utilizados mediante prehensión manual. De esta manera, se trataría de una "economía de materias primas", en los términos planteados por Perlès (1991) donde existen distintas cadenas operativas en diferentes materias primas dedicadas a la producción de instrumentos específicos. Se considera este caso como típico de ocupaciones sedentarizadas por la planificación y la inversión tecnológicas requeridas por estas cadenas operativas separadas y realizadas en distintas rocas (Perlès, 1991). La presencia de una trayectoria destinada a la producción de herramientas de procesamiento sobre rocas locales indicaría actividades domésticas en el contexto de una base residencial. En este sentido, la diversidad artefactual advertida, así como la presencia de determinados instrumentos (e.g. buriles y perforadores), es comparable con las observaciones realizadas en sitios relativamente contemporáneos en Antofagasta de la Sierra, como Casa Chávez Montículos, Las Escondidas 4 y Punta de la Peña 9, donde se infirieron actividades domésticas por parte de grupos familiares en el marco de ocupaciones residenciales (Escola, Hocsman y López Campeny, 2014; Sentinelli, 2020). 
La muestra cerámica analizada se caracteriza por pastas producidas con antiplásticos compatibles con las litologías de la Serranía de Coyahuaina y con aquellas presentes en el ámbito puneño en general. Al mismo tiempo, un porcentaje minoritario de pastas contiene granos blanquecinos (blandos) -las micacitas - fracción arena gruesa a gránulo fino, un tipo litológico ausente en la cuenca estudiada (las micacitas ordovícicas son grises y resistentes). Estos antiplásticos podrían ser el resultado de la cocción de arcillas o arcillitas blancas; por ejemplo, las descritas para la Fm. Tafna. Sería el caso de los fragmentos de pipa y del vaso de paredes finas cuyas pastas contienen escaso porcentaje de dichas micacitas blandas. Se propone que esta alfarería pudo haber sido manufacturada en lugares alejados, fuera de la cuenca del río Barrancas. Se debe destacar que materiales similares han sido descritos en pastas de cerámica manufacturada en otras regiones, situadas frecuentemente más al norte que la cuenca del río Barrancas; por ejemplo, el material identificado como micacitas blancas en pastas de la cerámica tardía registrada en la región de Casabindo, y también más al norte, en la región de la Laguna Pozuelos (ver Solá, 2011).

Los tiestos decorados y los fragmentos de pipa representan estilos y artefactos hasta ahora no reconocidos en el resto de la cuenca Barrancas; el tiesto AM9 tiene similitud decorativa, como hemos dicho, con el estilo Vaquerías de amplia dispersión en el NOA y, en Jujuy, fue determinado en Coch39 y Cerro Colorado, y también en Cobres (Puna de Salta) (Bugliani y Domingorena, 2012) con cronologías compatibles con la de Antigal Motaite. Por el momento, en Antigal Motaite, se carece de evidencias que indiquen alfarería manufacturada localmente, aunque tampoco se descarta. Del análisis comparativo con la alfarería de Alero Morro Blanco ${ }^{3}$ surge que, a pesar de las diferencias cronológicas, ambos sitios aportaron tiestos con pastas comunes y recurrentes a lo largo del tiempo. Esto denotaría continuidad en la "manera de hacer" las pastas y también podría estar marcando la naturaleza "local/regional" de los antiplásticos. Aunque las pastas de la cerámica ordinaria no presentan grandes diferencias entre ambos sitios, en Antigal Motaite predominan las tonalidades rojizas a diferencia de la cerámica de Alero Morro Blanco, que presenta colores amarronados y verdosos. En cambio, la cerámica decorada señala discrepancias entre ambos sitios en lo referente a los atributos formales, como ser formas y funcionalidad, espesores, colores y técnicas decorativas, hecho esperable debido al lapso registrado entre ambas ocupaciones.

Respecto de los fragmentos de pipa, es llamativo el diámetro calculado para los tres conductos (ca. $30 \mathrm{~mm}$ ). Se ha reconocido que el diámetro de las pipas para fumar consignadas en sitios del NOA tiene entre 9 y $13 \mathrm{~mm}$; esas pipas han tenido gran distribución regional y han sido utilizadas como marcadores de momentos tempranos relacionados principalmente con la tradición San Francisco (Dougherty, 1972). Numerosos autores han investigado la variedad y el uso de diversos tipos de pipas (Gili, Echeverría, Stovel, Deibel y Niemeyer, 2017; Lema et al., 2015) concluyendo que las mismas sirvieron además como sahumerios, especialmente, aquellas con diámetros mayores, como pudo haber sido el caso de los fragmentos de artefactos encontrados en Antigal Motaite. A la vez, la presencia de tiestos de grosor igual o mayor a $10 \mathrm{~mm}$ podría corresponderse con vasijas destinadas al almacenaje, hipótesis por ahora no comprobable a partir de la evidencia actualmente disponible.

Hasta la fecha, no se ha hallado en el área ningún otro sitio con alfarería contemporánea a Antigal Motaite (ca. 1700-1800 años AP). Hay otros tres sitios puneños - Coch 39 o Torre, Huáncar y Matancillas - con alfarería temprana pintada y grabada cuyas cronologías serían compatibles con los registros de Antigal Motaite, pero la cerámica recuperada no presenta rasgos comunes, excepto la presencia de los tres fragmentos 
de pipa, artefactos que también fueron hallados en esos tres sitios. Torre $(17 \mathrm{~km}$ al sudoeste de AM) es un sitio con ocupaciones que datan ca. 1800 AP (Fernández Distel, 2001), Huáncar (60 km al sudoeste) tiene ocupaciones datadas en ca. 2000 AP (Fernández Distel, 2006) así como Matancillas (70 km al sur) (Muscio, 2004). Las pastas de la cerámica de Matancillas parecen contener el mismo tipo de antiplásticos (Muscio, 2004) que las de Antigal Motaite y Alero Morro Blanco (Yacobaccio et al., 2020). Cabe señalar que Matancillas se ubica en la quebrada homónima de la Serranía de Cobres, caracterizada por las unidades metasedimentarias precámbricas a ordovícicas (Seggiaro et al., 2015). Por tal motivo, si en ambas zonas se manufacturó cerámica en lapsos equivalentes, son esperables pastas con antiplásticos similares en vasijas que bien podrían caracterizarse por formas, tamaños y colores diferentes como parecería ser la relación existente entre alfarería de Antigal Motaite y la de Matancillas. Como fue mencionado en otras ocasiones (Solá, 2011), es dificultoso distinguir cerámica local de alóctona cuando se trata de la "uniformidad y extensión" litológica de la Puna jujeño-salteña. Este es un rasgo geoambiental que introduce "ruido" en el análisis porque las pastas producidas en la región y en sectores aledaños podrían contener antiplásticos derivados de dichas formaciones, lo que lleva a establecer altas correlaciones, independientemente de los lugares y del momento de obtención de las materias primas. A la vez, la presencia de arcillitas blancas en las pastas (las micacitas blandas), conjuntamente con gránulos redondeados de cuarzo y de plagioclasa, estaría denotando alfarería manufacturada fuera del área de estudio, quizás en regiones alejadas como podrían ser las cuencas del norte de la Puna jujeña.

Los resultados presentados en este trabajo permitieron iniciar el desarrollo de algunos ejes de investigación. A partir de las evidencias de cerámica y lítico de Antigal Motaite, se puede proponer que el sitio integró redes de intercambio de larga distancia abastecidas por el tráfico caravanero. En otras localidades, se planteó que el intercambio a larga distancia, la constitución de redes de interacción y la circulación de bienes fue constante e involucró amplios recorridos durante el Temprano (Nielsen, 2007; Yacobaccio, 2012).

La cuenca de Barrancas habría sido propicia para el desarrollo del pastoreo y posiblemente de la agricultura, con condiciones más húmedas e indicadores de perturbación antrópica probablemente vinculados con el primero (Morales et al., 2018; Pirola et al., 2018). La presencia en el sitio de estructuras arquitectónicas y de cerámica gruesa podría estar relacionada con el almacenamiento estacional, en el marco de una estabilización de las poblaciones en las primeras aldeas y, más particularmente, con el impacto de la agricultura en las estrategias de subsistencia para este período. En efecto, la interacción entre pastoreo y agricultura se documentó en varias áreas, como Catamarca (Grant y Olivera, 2016), y debió resultar importante para poder sostener el desarrollo de las aldeas tempranas. La interacción entre las dos estrategias mencionadas al principio de este artículo (guano utilizado como abono o producción agrícola utilizada como forraje) aún no es visible en el sitio, pero podrá ser tema de futuros análisis.

Las cadenas operativas y las estructuras del sitio pudieron resultar de innovaciones tecnológicas concomitantes con el desarrollo de las poblaciones y el afianzamiento de la circulación de bienes, personas y conocimientos en torno a las aldeas. En términos generales, el conjunto de cadenas operativas del sitio correspondería a una organización social y económica alrededor de una subsistencia basada en el pastoreo y la agricultura, cada vez más estrechamente vinculadas. Más particularmente, debemos destacar el rol estratégico de algunas trayectorias tecnológicas en el marco de las actividades productivas y domésticas, tal como lo indican las puntas de proyectil, la diversidad de instrumentos relacionados con actividades domésticas de procesamiento, la presencia conjunta de pipas y de cerámica ordinaria, cada una con su propia trayectoria 
tecnológica. La evidencia actualmente disponible en Antigal Motaite es insuficiente para cuestionar el control social sobre las tareas tecnológicas y la articulación de las fuerzas y relaciones de producción (Lemonnier, 2010). Sin embargo, la especificidad de las cadenas operativas evidenciadas, en particular la notable "economía de materias primas" (sensu Perlès, 1991) y la naturaleza del sitio, incentivan una discusión acerca de la organización social vigente en las aldeas tempranas puneñas, la relación entre los distintos núcleos domésticos y la tecnología, el caravaneo y las estrategias de subsistencia (pastoreo y agricultura). Esta problemática puede constituir un eje que guiará las futuras investigaciones en la cuenca de Barrancas y, más específicamente, en Antigal Motaite.

\section{Agradecimientos}

A Santiago Lamas, Olga Puca y Dominga Puca. A las comunidades del Sianzo y de Barrancas. A Teresa Flores, Martín Alejo, a la municipalidad de Barrancas y a todos los integrantes del Proyecto Barrancas. A Roberto Asta y a la Cátedra de Sedimentología de la Facultad de Ciencias Exactas y Naturales de la Universidad de Buenos Aires. A Michael Glascock y a la Universidad de Missouri. Este trabajo se realizó en el marco de la Mission Archéologique Française en Argentine (Ministère de l'Europe et des Affaires Etrangères) y de diferentes proyectos UBACyT (F157 (2011-2014); 230BA (2014-2017); 399BA (2017-2019), del CONICET (PIP-0569, 2014-2017) y con la colaboración del VICAM (CONICET). Asimismo, agradecemos a los evaluadores y al comité editorial de la revista, cuyos comentarios y sugerencias contribuyeron a mejorar el trabajo. 


\section{Referencias citadas}

» Albeck, M. E. (2010). Estudios sobre agricultura prehispánica en Casabindo. En. M. A. Korstanje y M. N. Quesada (Eds.), Arqueología de la agricultura. Casos de estudio en la región andina argentina (pp. 12-47). San Miguel de Tucumán: Ediciones Magna.

» Albeck, M. E. y Zaburlín, M. A. (2008). Aportes a la cronología de los asentamientos agropastoriles de la Puna de Jujuy. Relaciones de la Sociedad Argentina de Antropología, XXXIII, 155-180. http://www.saantropologia.com.ar/textos/aportes-a-la-cronologia-de-losasentamientos-agropastoriles-de-la-puna-de-jujuy/ (Acceso: 30 de marzo, 2021).

» Aschero, C. (1979). Un asentamiento acerámico en la Quebrada de Inca Cueva (Jujuy). Informe preliminar sobre el sitio Inca Cueva cueva 4. En Actas I Jornadas de Arqueología del noroeste argentino (pp. 159-183). Buenos Aires: Universidad de San Salvador.

»Aschero, C. A. y Hocsman, S. (2011). Arqueología de las Ocupaciones Cazadoras-Recolectoras de fines del Holoceno medio de Antofagasta de la Sierra (Puna Meridional argentina). Chungara. Revista de Antropología Chilena, 43(1), 393-411. http://dx.doi.org/10.4067/S071773562011000300005

» Babot, M. del P. (2011). Cazadores-recolectores de los Andes Centro-Sur y procesamiento vegetal. Una discusión desde la Puna Meridional Argentina (ca. 7000-3200 años A.P.). Chungara. Revista de Antropología Chilena, 43(1), 413-432. http://dx.doi.org/10.4067/S071773562011000300006

» Boëda, E. (2013). Techno-logique et technologie. Une paléo-histoire des objets lithiques tranchants. Pringonrieux: @rchéo-éditions.com.

» Bugliani, M. F. y Domingorena, L. P. (2012). Una aproximación estilístico-tecnológica a la cerámica polícroma 'Vaquerías' del Noroeste Argentino. Estudios Atacameños, 43, 121-138. http://dx.doi.org/10.4067/S0718-10432012000100007

» Buitagro, L. G. y Larran, M. T. (1991). El clima de la Provincia de Jujuy. Jujuy: Facultad de Ciencias Agrarias-UNJu.

» Cartagena, I., Núñez, L. y Grosjean, M. (2007). Camelid domestication on the western slope of the Puna de Atacama, northern Chile. Anthropozoologica, 42(2), 155-173. https://sciencepress. $\mathrm{mnhn} . \mathrm{fr} / \mathrm{en} /$ periodiques/anthropozoologica/42/2/la-domestication-des-camelides-sur-leversant-occidental-de-la-puna-de-atacama-chili-du-nord (Acceso: 30 de marzo, 2021).

»De Feo, M. E. (2015). Una puesta al día sobre el Formativo de la Quebrada del Toro (Salta, Argentina). En M. A. Korstanje, M. Lazzari, M. Basile, F. Bugliani, V. Lema, L. Pereyra Domingorena y M. Quesada (Eds.), Crónicas materiales precolombinas. Arqueología de los primeros poblados del Noroeste argentino (pp. 277-311). Buenos Aires: Sociedad Argentina de Antropología.

» Delfino, D., Espiro, V. y Díaz, A. (2007). Excentricidad de las periferias: La región puneña de Laguna Blanca y las relaciones económicas con los valles mesotermales durante el primer milenio. En A. Nielsen, C. Rivolta, V. Seldes, M. Vázquez, y P. Mercolli (Eds.), Producción y circulación prehispánicas de bienes en el sur andino (pp. 167-190). Córdoba: Editorial Brujas.

» Dougherty, B. (1972). Las pipas de fumar arqueológicas de la Prov. de Jujuy. Consideraciones preliminares. Relaciones de la Sociedad Argentina de Antropología, VI, 83-89. http://www. saantropologia.com.ar/textos/las-pipas-de-fumar-arqueologicas-de-la-provincia-de-jujuy/ (Acceso: 30 de marzo, 2021).

»Escola, P. (2002). Caza y pastoralismo: un reaseguro para la subsistencia. Relaciones de la Sociedad Argentina de Antropología, XXVII, 233-246. http://www.saantropologia.com. ar/textos/caza-y-pastoralismo-un-reaseguro-para-la-subsistencia/ (Acceso: 30 de marzo, 2021). 
»Escola, P. S., Hocsman, S. y Babot, M. P. (2013). Entre las residencias y los campos de cultivo. Aportes de los cuchillos/raederas de módulo grandísimo a la cuestión del laboreo agrícola en Antofagasta de la Sierra (Puna de Atacama) durante el primer milenio d.C. Relaciones de la Sociedad Argentina de Antropología, XXXVIII(1), 83-110. http://www.saantropologia.com. ar/textos/entre-las-residencias-y-los-campos-de-cultivo-aportes-de-los-cuchillosraederas-demodulo-grandisimo-a-la-cuestion-del-laboreo-agricola-en-antofagasta-de-la-sierra-puna-decatamarca-durante-el-prim/ (Acceso: 30 de marzo, 2021).

»Escola, P. S., Hocsman, S. y López Campeny, S. (2014). Artefactos Líticos y Variabilidad de Asentamientos en Contextos Agro-Pastoriles de Antofagasta de la Sierra (Catamarca, Argentina). En P. Escola y S. Hocsman (Eds.), Artefactos Líticos, Movilidad y Funcionalidad de Sitios en Sudamérica: Problemas y Perspectivas (pp. 41-57). Oxford: BAR Internacional Series.

» Fernández Distel, A. (1998). Arqueología del Formativo en la Puna Jujeña 1800 ac. al 650 dc. Buenos Aires: CAEA.

» Fernández Distel, A. (2001). Catálogo del arte rupestre: Jujuy y su región. Buenos Aires: Dunken.

» Fernández Distel, A. (2006). Una aldea formativa en el altiplano argentino. Primera excavación en Huancar-Piscuno (Jujuy, Argentina). Revista andina, 42, 197-217.

» Gili, F., Echeverría, J., Stovel, E., Deibel, M. y Niemeyer, H. M. (2017). Las pipas del salar de Atacama: reevaluando su origen y uso. Estudios Atacameños, 54, 37-64. https://scielo.conicyt. cl/scielo.php?script=sci_arttext\&pid=S0718-10432017000100003 (Acceso: 30 de marzo, 2021).

» González, R. (2004). La arqueología del noroeste argentino y las culturas formativas de la cuenca del Titicaca. Relaciones de la Sociedad Argentina de Antropología, XXIX, 7-38. http:// www.saantropologia.com.ar/textos/la-arqueologia-del-noroeste-argentino-y-las-culturasformativas-de-la-cuenca-del-titicaca/ (Acceso: 30 de marzo, 2021).

» Grant, J. y Olivera, D. E. (2016). Isótopos estables, movilidad y camélidos en sociedades agro-pastoriles tempranas de la Puna Meridional Argentina. Arqueología, 22(Dossier), 13-35. https://doi.org/10.34096/arqueologia.t22.n0.3275

» Hogg, A. G, Heaton, T. J, Hua, Q., Palmer, J. G., Turney, C. S. M., Southon, J., Bayliss, A., Blackwell, P. G, Boswijk, G., Bronk Ramsey, C., Pearson, C., Petchey, F., Reimer, P., Reimer, R. y Wacker, L. (2020). SHCal20 Southern Hemisphere calibration, 0-55,000 years cal BP. Radiocarbon, 62(4), 759-778. https://doi.org/10.1017/RDC.2020.59

» Hoguin, R. y Kohan, P. (2018). Technological change during final Mid-Holocene and early Late Holocene (ca. 5000-2000 yrs. BP) in Barrancas (Jujuy province, Argentina). Journal of Lithic Studies, 5(2). https://doi.org/10.2218/jls.2801

» Inizan, M. L., Reduron, M., Roche, H. y Tixier, J. (1995). Technologie de la pierre taillée (Volumen 4). Meudon: CREP.

» Kohan, P. (2018). Tecnología lítica y petroglifos en Barrancas, Jujuy. (Tesis de Licenciatura inédita), Universidad de Buenos Aires, Argentina.

» Korstanje, A. (2007). Territorios Campesinos: Producción, Circulación y Consumo en los Valles Altos. En A. Nielsen, C. Rivolta, V. Seldes, M. Vázquez, P. Mercolli (Eds.), Producción y circulación prehispánicas de bienes en el sur andino (pp. 191-223). Córdoba: Editorial Brujas.

» Korstanje, M. A., Quesada, M., Franco Salvi, V., Lema, V. y Maloberti, M. (2015). Gente, tierra, agua y cultivos: Los primeros paisajes agrarios del Noroeste argentino. En M. A. Korstanje, M. Lazzari, M. Basile, F. Bugliani, V. Lema, L. Pereyra Domingorena y M. Quesada (Eds.), Crónicas materiales precolombinas. Arqueología de los primeros poblados del Noroeste argentino (pp. 721-749). Buenos Aires: Sociedad Argentina de Antropología.

» Lema, V. S., Andreoni, D., Capparelli, A., Ortiz, G., Spano, R., Quesada, M. y Zorzi, F. (2015). Protocolos y avances en el estudio de residuos de pipas arqueológicas de Argentina. Aportes para el entendimiento de metodologías actuales y prácticas pasadas. Estudios Atacameños, 51, 77-97. http://dx.doi.org/10.4067/S0718-10432015000200006

» Lemonnier, P. (1986). The study of material culture today: Towards an anthropology of technical systems. Journal of Anthropological Archaeology, 5, 147-186. https://doi.or g/10.1016/0278-4165(86)90012-7 
» Lemonnier, P. (2010). L'Etude des systèmes techniques. Une urgence en technologie culturelle. Technique et Culture, 54-55, 46-67. https://doi.org/10.4000/tc.1038

» López, G. E. (2008). Arqueología de Cazadores y Pastores en Tierras Altas: Ocupaciones humanas a lo largo del Holoceno en Pastos Grandes, Puna de Salta, Argentina. Oxford: BAR International Series.

》 Morales, M. (2011). Arqueología ambiental del Holoceno temprano y medio en la Puna Seca argentina. Modelos paleoambientales multi-escalas y sus implicancias para la Arqueología de Cazadores-Recolectores. Oxford: BAR International Series.

» Morales, M. R., Busto, S., Oxman, B. I., Pirola, M., Tchilinguirian, P., Orgeira, M. J. y Yacobaccio, H. D. (2018). Exploring habitat diversity of mid-holocene hunter-gatherers in the SouthCentral Andes: Multi-proxy analysis of Cruces Core 1 (TC1), Dry Puna of Jujuy, Argentina. Journal of Archaeological Science: Reports, 18, 708-721. https://doi.org/10.1016/j. jasrep.2017.07.010

» Muscio, H. (2004). Dinámica poblacional y evolución durante el período agroalfarero temprano en el Valle de San Antonio de los Cobres, Puna de Salta, Argentina. (Tesis Doctoral inédita), Universidad de Buenos Aires, Argentina. http://repositorio.filo.uba.ar/handle/ filodigital/1256

» Muscio, H. J. (2011). Ocupaciones humanas a cielo abierto de finales del Holoceno medio y comienzos del Holoceno tardío en el Valle de San Antonio de los Cobres, Puna de Salta. Comechingonia, 15, 171-190. https://doi.org/10.37603/2250.7728.v15.n1.17956

» Muscio, H. J. (2012). Modelling demographic dynamics and cultural evolution: The case of the early and mid-Holocene archaeology in the highlands of South America. Quaternary International, 256, 19-26. https://doi.org/10.1016/j.quaint.2011.10.021

» Muscio, H. y López, G. (2016). Radiocarbon dates and anthropogenic signal in the SouthCentral Andes (12,500-600 cal. years BP). Journal of Archaeological Science, 65, 93-102. https://doi.org/10.1016/j.jas.2015.11.007

» Nielsen, A. E. (2007). El Período de Desarrollos Regionales en la Quebrada de Humahuaca: aspectos cronológicos. En V. I. Williams, B. N. Ventura, A. B. Callegari y H. D. Yacobaccio (Eds.), Sociedades Precolombinas Surandinas: temporalidad, interacción y dinámica cultural (pp. 235-251). Buenos Aires: Taller internacional de arqueología del NOA y Andes Centro Sur.

» Núñez, L. A., Cartagena, I., Carrasco, C., López Mendoza, P., Rivera, F., De Souza, P., Santander, B. y Loyola, R. (2016). Nuevas Excavaciones en Tulán-54: Revelando la Arquitectura Ceremonial durante el Formativo Temprano de la Puna de Atacama. Revista Chilena de Antropología, 34, 65-79. https://revistas.uchile.cl/index.php/RCA/article/view/45149/47230 (Acceso: 30 de marzo, 2021).

» Núñez, L. y Dillehay, T. (1979). Movilidad Giratoria, Armonía Social y Desarrollo en los Andes Meridionales: Patrones de Tráfico e Interacción Económica. Antofagasta: Universidad Católica del Norte.

» Núñez, L. y Perlès, C. (2018). Ceremonialismo y congregación durante la transición arcaico tardío y formativo temprano en la circumpuna de Atacama (norte de Chile). Revista de Geografía Norte Grande, 70, 183-209. http://dx.doi.org/10.4067/S0718-34022018000200183

»Oliszewski, N. y Olivera, D. E. (2009). Variabilidad racial de macrorrestos arqueológicos de Zea mays (Poaceae) y sus relaciones con el proceso agropastoril en la Puna meridional argentina (Antofagasta de la Sierra, Catamarca). Darwiniana, 47(1), 76-91. https://doi.org/10.14522/ darwiniana.2014.471.42

» Olivera, D. (2012). El Formativo en los Andes del Sur: La incorporación de la opción productiva. En M. T. de Haro, A. M. Rocchietti, M. A. Runcio, O. Hernández de Lara y M. V. Fernández (Eds.), Interculturalidad y Ciencias. Experiencias desde América Latina (pp. 15-49). Buenos Aires: Centro de Investigaciones Precolombinas.

» Olivera, D. E., Elías, A. M., Pérez, M. y Salminci, P. (2015). Corral Grande 1 y Arroyo Seco: nuevos aportes al Formativo de Antofagasta de la Sierra (Provincia de Catamarca, Puna meridional argentina). Comenchingonia, 19, 37-66. https://doi.org/10.37603/2250.7728.v19.n1.27370 
» Oxman, B. I., Pirola, M., Bustos, S., Morales, M. R., Tchilinguirian, P. y Orgeira, M. J. (2019). Environmental trends between 2400 and 1200 BP in Barrancas, Argentinean Puna: Impacts on local resource variability and socioeconomic organization. Geoarchaeology, 35 (2), 243-257. https://doi.org/10.1002/gea.21767

"Perlès, C. (1991). Economie des matières premières et économie du débitage: deux conceptions opposées. En 25 ans d'Etudes Technologiques en Préhistoire. Xle Rencontres Internationales d'Archéologie et d'Histoire d'Antibes (pp. 35-45). Juan-les-Pins: Editions APDCA.

»Pirola, M., Bustos, S., Morales, M. R., Orgeira, M. J., Oxman, B. I., Tchilinguirian, P. y Vázquez, C. (2018). The mid to late Holocene transition in Barrancas, Jujuy, Argentina: Regional climate change, local environments and archaeological implications. Journal of Archaeological Science: Reports, 18, 722-738. https://doi.org/10.1016/j.jasrep.2017.06.019

»Quesada, M. N. (2010). Agricultura campesina en el área de Antofalla. En M. A. Korstanje y M. N. Quesada (Eds.), Arqueología de la agricultura. Casos de estudio en la región andina argentina (pp. 76-103). San Miguel de Tucumán: Ediciones Magna.

» Raffino, R. (1977). Las aldeas del Formativo inferior en la Quebrada del Toro, (Pcia. de Salta. Argentina). Obra del Centenario del Museo de La Plata, II, 253-299. https://doi.org/10.22199/ S07181043.1977.0005.00007

» Rubiolo, D., Seggiaro, R., Gallardo, E., Disalvo, A., Sánchez, M., Turel, A., Ramallo, E., Sandruss, A. y Godeas, M. (2003). Hoja Geológica 2366-II / 2166-IV, La Quiaca. Provincias de Jujuy y Salta (Boletín 246). Buenos Aires: Instituto de Geología y Recursos Minerales, Servicio Geológico Minero Argentino. https://repositorio.segemar.gov.ar/handle/308849217/146\#: :text=La\%20 HOJA\%202366\%2DII\%20y,Cordillera\%20Oriental\%20y\%20Sierras\%20Subandinas.

»Salazar, J. y Franco Salvi, V. (2015). Producción y reproducción social durante el primer milenio en el valle de Tafí. En M. A. Korstanje, M. Lazzari, M. Basile, F. Bugliani, V. Lema, L. Pereyra Domingorena y M. Quesada (Eds.), Crónicas materiales precolombinas. Arqueología de los primeros poblados del Noroeste argentino (pp. 81-110). Buenos Aires: Sociedad Argentina de Antropología.

»Seggiaro, R. E., Becchio, R., Bercheñi, V. y Ramallo, L. (2015). Hoja geológica 2366-III Susques, provincias de Jujuy y Salta (Boletín 414). Buenos Aires: Instituto de Geología y Recursos Minerales, Servicio Geológico Minero Argentino. https://repositorio.segemar.gov.ar/ handle/308849217/185

» Sentinelli, N. (2020). Tecnología lítica y variabilidad en la puna meridional argentina durante el primer milenio de la era. El caso de Las Escondidas 4 (LES 4). (Tesis doctoral inédita), Universidad Nacional de Córdoba, Argentina.

»Solá, P. (2011). Tiestos trazadores de cerámica exótica en sitios tardíos de los alrededores de Susques (Jujuy, Puna argentina). Intersecciones en Antropología, 12, 177-194. http://www. ridaa.unicen.edu.ar/xmlui/handle/123456789/1321

» Stuiver, M. y Reimer, P. J. (1993). Extended 14C data base and revised Calib 3.0 14C age calibration program. Radiocarbon, 35(1), 215-230. https://doi.org/10.1017/ S0033822200013904

" Tarragó, M. (1984). La historia de los pueblos circumpuneños en relación con el altiplano y los Andes Meridionales. Estudios Atacameños, 7, 93-104. https://doi.org/10.22199/ S07181043.1984.0007.00010

" Tchilinguirian, P. y Olivera, D. E. (2010). Agricultura, ambiente y sustentabilidad agrícola en el desierto: el caso de Antofagasta de la Sierra. En M. A., Korstanje y M. N. Quesada (Eds.), Arqueología de la agricultura. Casos de estudio en la región andina argentina (pp. 104-129). San Miguel de Tucumán: Ediciones Magna.

»Yacobaccio, H. D. (1994). Biomasa animal y consumo en el Pleistoceno-Holoceno Surandino. Arqueología, 4, 43-71.

» Yacobaccio, H. D. (2012). Intercambio y caravanas de llamas en el sur andino (3000-1000 AP). Comechingonia, 16, 31-51. https://doi.org/10.37603/2250.7728.v16.n1.17960 
» Yacobaccio, H. D., Escola, P. S., Pereyra, F. X., Lazzari, M. y Glascock, M. D. (2004). Quest for ancient routes: obsidian sourcing research in Northwestern Argentina. Journal of Archaeological Science, 31, 193-204. https://doi.org/10.1016/j.jas.2003.08.001

» Yacobaccio, H., Catá, P., Morales, M., Solá, P., Alonso, S., Rosenbuch, M., Vázquez, C., Samec, C., Oxman, B. y Cáceres, M. (2011). El uso de cuevas por pastores andinos: El caso de cueva Quispe (Susques, Puna de Jujuy). En G. López y H. Muscio (Eds.), Arqueología de la Puna argentina: Perspectivas actuales en el estudio de la diversidad y el cambio cultural (pp. 33-47). Oxford: BAR International Series.

» Yacobaccio, H. D., Hoguin, R., Solá, P., Oxman, B., Rouan Sirolli, M. y Kohan, P. (2020). Ocupaciones tempranas (ca. 3200-2200 años AP) en el Alero del Morro Blanco (Barrancas, Pcia. de Jujuy, Agentina): Resultados preliminares. Estudios Sociales del NOA, (21), 21-52. http://revistascientificas.filo.uba.ar/index.php/esnoa/article/view/8893 (Acceso: 30 de marzo, 2021).

»Zappettini, E. (1989). Geología y metalogénesis de la región comprendida entre las localidades de Santa Ana y Cobres, provincias de Jujuy y Salta, República Argentina. (Tesis Doctoral inédita), Universidad de Buenos Aires, Argentina. http://hdl.handle.net/20.500.12110/tesis_ n2236_Zappettini 